\title{
The period function of Hamiltonian systems with separable variables
}

\author{
Jordi Villadelprat and Xiang Zhang \\ Departament d'Enginyeria Informàtica i Matemàtiques, ETSE, \\ Universitat Rovira i Virgili, 43007 Tarragona, Spain \\ Email: jordi.villadelprat@urv.cat \\ School of Mathematical Sciences, MOE-LSC, Shanghai Jiao Tong University, \\ Shanghai 200240, People's Republic of China \\ Email:xzhang@sjtu.edu.cn
}

February 20, 2019

\begin{abstract}
In this paper we study the period function of those planar Hamiltonian differential systems for which the Hamiltonian function $H(x, y)$ has separable variables, i.e., it can be written as $H(x, y)=$ $F_{1}(x)+F_{2}(y)$. More concretely we are concerned with the search of sufficient conditions implying the monotonicity of the period function, i.e., the absence of critical periodic orbits. We are also interested in the uniqueness problem and in this respect we seek conditions implying that there exists at most one critical periodic orbit. We obtain in a unified way several sufficient conditions that already appear in the literature, together with some other results that to the best of our knowledge are new. Finally we also investigate the limit of the period function as the periodic orbits tend to the boundary of the period annulus of the center.
\end{abstract}

\section{Introduction and definitions}

The present paper deals with the class of planar Hamiltonian differential systems

$$
\left\{\begin{array}{l}
\dot{x}=-H_{y}(x, y), \\
\dot{y}=H_{x}(x, y),
\end{array}\right.
$$

where the Hamiltonian function has separable variables, i.e., it has the special form

$$
H(x, y)=F_{1}(x)+F_{2}(y) .
$$

For $i=1,2$ we suppose that $F_{i}(z)$ is an analytic function on $\mathbb{R}$ with a local minimum at $z=0$, so that

$$
\left\{\begin{array}{l}
\dot{x}=-F_{2}^{\prime}(y), \\
\dot{y}=F_{1}^{\prime}(x),
\end{array}\right.
$$

has a critical point at the origin of center type. Recall that a critical point $p$ of a planar differential system is a center if it has a punctured neighbourhood that consists entirely of periodic orbits surrounding $p$. The period annulus is the largest punctured neighbourhood with this property and we shall denote it by $\mathscr{P}$.

Keywords: Hamiltonian differential system, center, period function, critical periodic orbit.

2010 MSC: 34C25, 37J35, 70H05, 70K42, 70K70. 
The solution curves of (1) are inside the energy levels of $H$. We can assume without loss of generality that $F_{i}(0)=0$ and, accordingly, $H(0,0)=0$. Thus $H(\mathscr{P})=\left(0, h_{0}\right)$ for some $h_{0} \in \mathbb{R}_{>0} \cup\{+\infty\}$. It is easy to see on the other hand that the energy level of the Hamiltonian parametrizes the set of periodic orbits in $\mathscr{P}$. Hence for each $h \in\left(0, h_{0}\right)$ we denote by $\gamma_{h}$ the periodic orbit of $\mathscr{P}$ inside the energy level $H=h$.

We are concerned with the period function of the center, which assigns to each periodic orbit in $\mathscr{P}$ its period. In order to study it we consider $h \longmapsto T(h):=$ period of $\gamma_{h}$, that can be written as

$$
T(h)=\int_{\gamma_{h}} \frac{d x}{F_{2}^{\prime}(y)} \text { for each } h \in\left(0, h_{0}\right) .
$$

(Here, and in what follows, we take the oval $\gamma_{h}$ clockwise oriented.) This is an analytic map that provides the qualitative properties of the period function that we are interested in. Particularly the existence of critical periods, which are isolated critical points of this function, i.e., those values $\hat{h} \in\left(0, h_{0}\right)$ such that $T^{\prime}(h)=\alpha(h-\hat{h})^{k}+\mathrm{o}\left((h-\hat{h})^{k}\right)$ with $\alpha \neq 0$ and $k \geqslant 1$. In this case we shall say that $\gamma_{\hat{h}}$ is a critical periodic orbit of multiplicity $k$ of the center. One can readily see that this definition does not depend on the particular parametrization of the set of periodic orbits in $\mathscr{P}$ used. We say that the period function of the center is monotonous increasing (respectively, decreasing) if $T^{\prime}(h)$ is strictly positive (respectively, negative) for all $h \in\left(0, h_{0}\right)$.

The problem of bounding the number of critical periodic orbits is analogous to the problem of bounding the number of limit cycles, which is related to the well known Hilbert's 16th Problem (see [1, 8, 26, 34] and references therein) and its various weakened versions. Questions related to the behaviour of the period function have been extensively studied by a number of authors. Let us quote for instance the problems of isochronicity [6, 17], monotonicity [2,30] or bifurcation of critical periodic orbits [5, 27].

The paper is organized as follows. In Section 2 we prove some auxiliary tools that will be used henceforth. More concretely, we first use the Gelfand-Leray derivation formula to obtain an expression for $T^{\prime}(h)$ given in terms of an Abelian integral, see Lemma 2.3. Next we prove a general result, namely Proposition 2.7, that enables us to write this type of Abelian integral more conveniently in order to take advantage of the involutions associated to the Hamiltonian. Section 3 is devoted to obtain sufficient conditions for the monotonicity of the period function. To this aim we begin by proving Proposition 3.2, which particularized yields to well-known monotonicity conditions, see Corollaries 3.4 and 3.5, that were previously obtained by other authors (see $[2,9,25,32]$ ). Proposition 3.7 is the main result in Section 3 and to the best of our knowledge it constitutes a new result. Section 4 is addressed to the problem of uniqueness of critical periodic orbits and in this regard we prove Theorems 4.1 and 4.11 , which provide conditions implying the existence of at most one critical periodic orbit. Finally Section 5 deals with the limit of the period function $T(h)$ as $h$ tends to the endpoints of its domain $\left(0, h_{0}\right)$, see Theorem 5.1. This has been studied previously by several authors under different settings (see $[4,7,15,18,31]$ and references therein). Our contribution, Theorem 5.1, is motivated by a property used by Kaplan and Yorke [13] in their proof on the existence of periodic solutions of differential-delay equations. Once we prove our result we will make some comments concerning a delicate point in their proof that we think did not receive the required attention, see Remark 5.2.

\section{Auxiliary results}

Let us fix that $F_{i}(z)=\alpha_{i} z^{k_{i}}+\mathrm{o}\left(z^{k_{i}}\right)$ for some even number $k_{i}$ and positive real number $\alpha_{i}$. In addition, let $I_{1}$ and $I_{2}$ denote, respectively, the projection of $\mathscr{P}$ on $y=0$ and $x=0$. Note that $I_{i}=\left(e_{i}^{-}, e_{i}^{+}\right)$with $e_{i}^{-}<0<e_{i}^{+}$and $z F_{i}^{\prime}(z)>0$ for all $z \in I_{i} \backslash\{0\}$. Each $F_{i}$ defines an analytic involution $\sigma_{i}$ on $I_{i}$ by means the relation

$$
F_{i}(z)=F_{i}\left(\sigma_{i}(z)\right) \text { for all } z \in I_{i}
$$

Recall that a function $\sigma$ is said to be an involution if $\sigma \circ \sigma=I d$ and $\sigma \neq I d$. In this respect observe that $\sigma_{i}(0)=0$ and $\sigma_{i}^{\prime}(z)<0$ for all $z \in I_{i}$. In the statement of our first result, and in what follows, the 
multiplicity of an analytic function $f$ at $x=\hat{x}$ is denoted by $\operatorname{mult}(f, \hat{x})$.

Lemma 2.1. Let $a$ be an analytic function on $I_{1}$ with mult $(a, 0) \geqslant k_{1}-1$ and let $\ell$ be a function such that $F_{2}^{\prime} \ell$ is analytic on $I_{2}$. Then

$$
\int_{\gamma_{h}} a(x) \ell(y) d x=\int_{\gamma_{h}}\left(\frac{a}{F_{1}^{\prime}}\right)^{\prime}(x) \hat{\ell}(y) d x, \text { where } \hat{\ell}^{\prime}=\ell F_{2}^{\prime} .
$$

Proof. Let $R(x)$ and $S(y)$ be analytic functions on $I_{1}$ and $I_{2}$, respectively. If $(x, y) \in \gamma_{h}$ then

$$
d(S(x) R(y))=S^{\prime}(x) R(y) d x+S(x) R^{\prime}(y) d y=S^{\prime}(x) R(y) d x-S(x) R^{\prime}(y) \frac{F_{1}^{\prime}(x)}{F_{2}^{\prime}(y)} d x
$$

and, consequently,

$$
\int_{\gamma_{h}} S^{\prime}(x) R(y) d x=\int_{\gamma_{h}}\left(S F_{1}^{\prime}\right)(x)\left(\frac{R^{\prime}}{F_{2}^{\prime}}\right)(y) d x .
$$

The result follows from this equality taking $a=S F_{1}^{\prime}$ and $\ell=\frac{R^{\prime}}{F_{2}^{\prime}}$, which leads to $S=\frac{a}{F_{1}^{\prime}}$ and $R^{\prime}=\ell F_{2}^{\prime}$.

Lemma 2.2. If $R$ and $S$ are analytic functions on $I_{1}$ and $I_{2}$, respectively, then

$$
\frac{d}{d h} \int_{\gamma_{h}} R(x) S(y) d x=\int_{\gamma_{h}} R(x)\left(\frac{S^{\prime}}{F_{2}^{\prime}}\right)(y) d x .
$$

Proof. This follows by the Gelfand-Leray derivation formula (see for instance [12, Theorem 26.32]), which asserts that

$$
\frac{d}{d h} \int_{\gamma_{h}} \omega=\int_{\gamma_{h}} \eta
$$

provided that $d \omega=d H \wedge \eta$.

In all over the paper for the sake of shortness we will use the notation

$$
\ell_{i}:=\frac{F_{i}}{F_{i}^{\prime}} \text { for } i=1,2 .
$$

Since $F_{i}(z)=\alpha_{i} z^{k_{i}}+\mathrm{o}\left(z^{k_{i}}\right)$, note that $\ell_{i}$ is an analytic function on $I_{i}=\left(e_{i}^{-}, e_{i}^{+}\right)$with $\ell_{i}(z)=\frac{1}{k_{i}} z+\mathrm{o}(z)$.

Lemma 2.3. The period function of the center at the origin of the differential system (1) verifies

$$
T^{\prime}(h)=\frac{1}{h} \int_{\gamma_{h}}\left(\ell_{1}^{\prime}(x)+\ell_{2}^{\prime}(y)-1\right) \frac{d x}{F_{2}^{\prime}(y)} .
$$

Proof. Note that, since $h=F_{1}(x)+F_{2}(y)$ for all $(x, y) \in \gamma_{h}$,

$$
h T(h)=\int_{\gamma_{h}} F_{1}(x) \frac{d x}{F_{2}^{\prime}(y)}+\int_{\gamma_{h}} F_{2}(y) \frac{d x}{F_{2}^{\prime}(y)}=\int_{\gamma_{h}}\left(\frac{F_{1}}{F_{1}^{\prime}}\right)^{\prime}(x) y d x+\int_{\gamma_{h}}\left(\frac{F_{2}}{F_{2}^{\prime}}\right)(y) d x,
$$

where in the second equality we applied Lemma 2.1. Then by Lemma 2.2 we can assert that

$$
(h T(h))^{\prime}=\int_{\gamma_{h}}\left(\frac{F_{1}}{F_{1}^{\prime}}\right)^{\prime}(x) \frac{d x}{F_{2}^{\prime}(y)}+\int_{\gamma_{h}}\left(\frac{F_{2}}{F_{2}^{\prime}}\right)^{\prime}(y) \frac{d x}{F_{2}^{\prime}(y)} .
$$

Thus

$$
h T^{\prime}(h)=\int_{\gamma_{h}}\left(\frac{F_{1}}{F_{1}^{\prime}}\right)^{\prime}(x) \frac{d x}{F_{2}^{\prime}(y)}+\int_{\gamma_{h}}\left(\frac{F_{2}}{F_{2}^{\prime}}\right)^{\prime}(y) \frac{d x}{F_{2}^{\prime}(y)}-\int_{\gamma_{h}} \frac{d x}{F_{2}^{\prime}(y)}
$$

and so the result follows. 
Taking advantage of the previous lemmas we can already obtain an expression that enables to study the monotonicity of the period function near a non-degenerate center.

Lemma 2.4. For $i=1,2$, suppose that $F_{i}(z)=\alpha_{i, 2} z^{2}+\alpha_{i, 3} z^{3}+\alpha_{i, 4} z^{4}+\mathrm{o}\left(z^{4}\right)$. Assume furthermore that $\Delta:=5\left(\alpha_{1,3}^{2}+\alpha_{2,3}^{2}\right)-4\left(\alpha_{1,2} \alpha_{1,4}+\alpha_{2,2} \alpha_{2,4}\right) \neq 0$. Then the period function is locally monotonous increasing (respectively, decreasing) near the center in case that $\Delta$ is positive (respectively, negative).

Proof. For $i=1,2$, let us define $a_{i}:=\ell_{i}^{\prime}-\frac{1}{2}$ for the sake of shortness. Then by Lemma 2.3 we can write

$$
h T^{\prime}(h)=\int_{\gamma_{h}} a_{1}(x) \frac{d x}{F_{2}^{\prime}(y)}+\int_{\gamma_{h}}\left(\frac{a_{2}}{F_{2}^{\prime}}\right)(y) d x=\int_{\gamma_{h}}\left(\frac{a_{1}}{F_{1}^{\prime}}\right)^{\prime}(x) y d x+\int_{\gamma_{h}}\left(\frac{a_{2}}{F_{2}^{\prime}}\right)(y) d x,
$$

where in the second equality we applied Lemma 2.1 taking $a_{1}(0)=0$ and $F_{1}^{\prime \prime}(0) \neq 0$ into account. Therefore, since $\left(\frac{a_{i}}{F_{i}^{\prime}}\right)(z)$ is analytic at $z=0$ for $i=1,2$, we can apply Green's Theorem (see [28]) to obtain

$$
h T^{\prime}(h)=\iint_{\operatorname{Int}(\gamma)}\left(\left(\frac{a_{1}}{F_{1}^{\prime}}\right)^{\prime}(x)+\left(\frac{a_{2}}{F_{2}^{\prime}}\right)^{\prime}(y)\right) d y d x
$$

where $\operatorname{Int}(\gamma)$ stands for the bounded connected component of $\mathbb{R}^{2} \backslash\{\gamma\}$. (We shall use this notation hereafter.) Now, since one can readily verify that $\left(\frac{a_{i}}{F_{i}^{\prime}}\right)(z)=-\frac{1}{4} \frac{\alpha_{i, 3}}{\alpha_{i, 2}^{2}}+\frac{3}{16} \frac{5 \alpha_{i, 3}^{2}-4 \alpha_{i, 2} \alpha_{i, 4}}{\alpha_{i, 2}^{3}} z+\mathrm{o}(z)$, the result follows.

Definition 2.5. Let $\sigma$ be an analytic involution and consider a given function $f$. The $\sigma$-even part of $f$ is

$$
\mathscr{P}_{\sigma}(f)(x):=\frac{f(x)-f(\sigma(x)) \sigma^{\prime}(x)}{2} .
$$

We say that $f$ is $\sigma$-even if $\mathscr{P}_{\sigma}(f)=f$.

Observe that this definition coincides with the usual notion of evenness in case that $\sigma=-I d$. Likewise we have the following characterization of $\sigma$-evenness.

Lemma 2.6. $f$ is $\sigma$-even if, and only if, there exits $g$ such that $f=\mathscr{P}_{\sigma}(g)$.

Proof. The necessity is obvious. In order to see the sufficiency, assume that $f(z)=\frac{1}{2}\left(g(z)-g(\sigma(z)) \sigma^{\prime}(z)\right)$ for some $g$. Then

$$
\begin{aligned}
2 \mathscr{P}_{\sigma}(f)(z) & =g(z)-g(\sigma(z)) \sigma^{\prime}(z)-\left(g(\sigma(z))-g\left(\sigma^{2}(z)\right) \sigma^{\prime}(\sigma(z))\right) \sigma^{\prime}(z) \\
& =g(z)-g(\sigma(z)) \sigma^{\prime}(z)-\left(g(\sigma(z))-\frac{g(z)}{\sigma^{\prime}(z)}\right) \sigma^{\prime}(z)=g(z)-g(\sigma(z)) \sigma^{\prime}(z)=2 f(z),
\end{aligned}
$$

which gives $\mathscr{P}_{\sigma}(f)=f$, as desired.

Proposition 2.7. Let $\gamma$ be an oval, clockwise oriented, inside the energy level $F_{1}(x)+F_{2}(y)=h$ and define

$$
C:=\int_{\gamma} a(x) b(y) \frac{d x}{F_{2}^{\prime}(y)}
$$

where $a$ and $b$ are analytic functions on $I_{1}=\left(e_{1}^{-}, e_{1}^{+}\right)$and $I_{2}=\left(e_{2}^{-}, e_{2}^{+}\right)$, respectively. Then, if we denote the first quadrant by $Q_{1}$, the following hold:

(a) $C=\int_{\gamma} \mathscr{P}_{\sigma_{1}}(a)(x) \mathscr{P}_{\sigma_{2}}(b)(y) \frac{d x}{F_{2}^{\prime}(y)}=4 \int_{Q_{1} \cap \gamma} \mathscr{P}_{\sigma_{1}}(a)(x) \mathscr{P}_{\sigma_{2}}(b)(y) \frac{d x}{F_{2}^{\prime}(y)}$ 
(b) $h C=4 \iint_{Q_{1} \cap \operatorname{Int}(\gamma)}\left(\mathscr{P}_{\sigma_{1}}\left(\left(a \ell_{1}\right)^{\prime}\right)(x) \mathscr{P}_{\sigma_{2}}(b)(y)+\mathscr{P}_{\sigma_{1}}(a)(x) \mathscr{P}_{\sigma_{2}}\left(\left(b \ell_{2}\right)^{\prime}\right)(y)\right) d y d x$.

Proof. Let us split the given oval as the concatenation $\gamma=\gamma_{1}+\gamma_{4}+\gamma_{3}+\gamma_{2}$, where $\gamma_{i}$ is the intersection of $\gamma$ with the $i$-th quadrant. Define $\psi_{1}(x, y):=\left(\sigma_{1}(x), y\right)$ and $\psi_{2}(x, y):=\left(x, \sigma_{2}(y)\right)$. Due to $\sigma_{i}^{\prime}(z)<0$ for all $z \in I_{i}$, note that $\psi_{1}$ and $\psi_{2}$ are analytic diffeomorphisms on $\mathscr{P}$ that reverse orientation. On account of this, and the fact that $\psi_{1}\left(\gamma_{2} \cup \gamma_{3}\right)=\gamma_{1} \cup \gamma_{4}$, the coordinate change $(x, y)=\psi_{1}(u, v)$ yields

$$
\int_{\gamma_{3}+\gamma_{2}} a(x) b(y) \frac{d x}{F_{2}^{\prime}(y)}=-\int_{\gamma_{1}+\gamma_{4}} a\left(\sigma_{1}(u)\right) b(v) \sigma_{1}^{\prime}(u) \frac{d u}{F_{2}^{\prime}(v)} .
$$

Hence, on account of Definition 2.5,

$$
\begin{aligned}
C & =\int_{\gamma} a(x) b(y) \frac{d x}{F_{2}^{\prime}(y)}=\int_{\gamma_{1}+\gamma_{4}} a(x) b(y) \frac{d x}{F_{2}^{\prime}(y)}+\int_{\gamma_{3}+\gamma_{2}} a(x) b(y) \frac{d x}{F_{2}^{\prime}(y)} \\
& =\int_{\gamma_{1}+\gamma_{4}}\left(a(x)-a\left(\sigma_{1}(x)\right) \sigma_{1}^{\prime}(x)\right) b(y) \frac{d x}{F_{2}^{\prime}(y)}=2 \int_{\gamma_{1}+\gamma_{4}} \mathscr{P}_{\sigma_{1}}(a)(x) b(y) \frac{d x}{F_{2}^{\prime}(y)} .
\end{aligned}
$$

On the other hand, since $\psi_{2}\left(\gamma_{4}\right)=\gamma_{1}$, the change of coordinates $(x, y)=\psi_{2}(u, v)$ yields

$$
\int_{\gamma_{4}} \mathscr{P}_{\sigma_{1}}(a)(x) b(y) \frac{d x}{F_{2}^{\prime}(y)}=-\int_{\gamma_{1}} \mathscr{P}_{\sigma_{1}}(a)(u) b\left(\sigma_{2}(v)\right) \frac{d u}{F_{2}^{\prime}\left(\sigma_{2}(v)\right)}=-\int_{\gamma_{1}} \mathscr{P}_{\sigma_{1}}(a)(u) b\left(\sigma_{2}(v)\right) \sigma_{2}^{\prime}(v) \frac{d u}{F_{2}^{\prime}(v)},
$$

where in the second equality we used that $F_{2}^{\prime}(y)=F_{2}^{\prime}\left(\sigma_{2}(y)\right) \sigma_{2}^{\prime}(y)$. Therefore, from (2),

$$
C=\int_{\gamma} a(x) b(y) \frac{d x}{F_{2}^{\prime}(y)}=2 \int_{\gamma_{1}} \mathscr{P}_{\sigma_{1}}(a)(x)\left(b(y)-b\left(\sigma_{2}(y)\right) \sigma_{2}^{\prime}(y)\right) \frac{d x}{F_{2}^{\prime}(y)}=4 \int_{\gamma_{1}} \mathscr{P}_{\sigma_{1}}(a)(x) \mathscr{P}_{\sigma_{2}}(b)(y) \frac{d x}{F_{2}^{\prime}(y)},
$$

and this proves $(a)$. Note in this respect that, thanks to the above equality, in the definition of $C$ we can replace $a$ by $\mathscr{P}_{\sigma_{1}}(a)$ and $b$ by $\mathscr{P}_{\sigma_{2}}(b)$ because, on account of Lemma 2.6, $\mathscr{P}_{\sigma}\left(\mathscr{P}_{\sigma}(f)\right)=\mathscr{P}_{\sigma}(f)$ for any $f$. Let us turn next to the proof of $(b)$. Due to $F_{1}(x)+F_{2}(y)=h$ for all $(x, y) \in \gamma$ we have

$$
h C=\int_{\gamma}\left(F_{1}(x)+F_{2}(y)\right) a(x) b(y) \frac{d x}{F_{2}^{\prime}(y)}=\int_{\gamma}\left(a F_{1}\right)(x)\left(\frac{b}{F_{2}^{\prime}}\right)(y) d x+\int_{\gamma} a(x)\left(\frac{b F_{2}}{F_{2}^{\prime}}\right)(y) d x .
$$

Thus by applying Lemma 2.1 we get

$$
h C=\int_{\gamma}\left(\frac{a F_{1}}{F_{1}^{\prime}}\right)^{\prime}(x) B(y) d x+\int_{\gamma} a(x)\left(\frac{F_{2} b}{F_{2}^{\prime}}\right)(y) d x=\int_{\gamma}\left(\left(a \ell_{1}\right)^{\prime}(x) B(y)+a(x)\left(b \ell_{2}\right)(y)\right) d x,
$$

where $B^{\prime}=b$, and then the application of Green's Theorem (see for instance [28]) yields

$$
h C=\iint_{\operatorname{Int}(\gamma)}\left(\left(a \ell_{1}\right)^{\prime}(x) b(y)+a(x)\left(b \ell_{2}\right)^{\prime}(y)\right) d y d x .
$$

Similarly as before, we split $\operatorname{Int}(\gamma)=\cup_{i=1}^{4} R_{i}$ where $R_{i}$ is the intersection of $\operatorname{Int}(\gamma)$ with the $i$-th quadrant. Since $\psi_{1}\left(R_{2} \cup R_{3}\right)=R_{1} \cup R_{4}$, the coordinate change $(x, y)=\psi_{1}(u, v)$ yields

$\iint_{R_{2} \cup R_{3}}\left(\left(a \ell_{1}\right)^{\prime}(x) b(y)+a(x)\left(b \ell_{2}\right)^{\prime}(y)\right) d y d x=-\iint_{R_{1} \cup R_{4}}\left(\left(a \ell_{1}\right)^{\prime}\left(\sigma_{1}(u)\right) b(v)+a(\sigma(u))\left(b \ell_{2}\right)^{\prime}(v)\right) \sigma_{1}^{\prime}(u) d v d u$

Therefore,

$$
h C=2 \iint_{R_{1} \cup R_{4}}\left(\mathscr{P}_{\sigma_{1}}\left(\left(a \ell_{1}\right)^{\prime}\right)(x) b(y)+\mathscr{P}_{\sigma_{1}}(a)(x)\left(b \ell_{2}\right)^{\prime}(y)\right) d y d x .
$$


On the other hand, since $\psi_{2}\left(R_{4}\right)=R_{1}$, the change of coordinates $(x, y)=\psi_{2}(u, v)$ yields

$$
\begin{aligned}
\iint_{R_{4}}\left(\mathscr{P}_{\sigma_{1}}\left(\left(a \ell_{1}\right)^{\prime}\right)(x) b(y)\right. & \left.+\mathscr{P}_{\sigma_{1}}(a)(x)\left(b \ell_{2}\right)^{\prime}(y)\right) d y d x \\
& =-\iint_{R_{1}}\left(\mathscr{P}_{\sigma_{1}}\left(\left(a \ell_{1}\right)^{\prime}\right)(u) b\left(\sigma_{2}(v)\right)+\mathscr{P}_{\sigma_{1}}(a)(u)\left(b \ell_{2}\right)^{\prime}\left(\sigma_{2}(v)\right)\right) \sigma_{2}^{\prime}(v) d v d u
\end{aligned}
$$

Finally taking Definition 2.5 into account once again we get

$$
h C=4 \iint_{R_{1}}\left(\mathscr{P}_{\sigma_{1}}\left(\left(a \ell_{1}\right)^{\prime}\right)(x) \mathscr{P}_{\sigma_{2}}(b)(y)+\mathscr{P}_{\sigma_{1}}(a)(x) \mathscr{P}_{\sigma_{2}}\left(\left(b \ell_{2}\right)^{\prime}\right)(y)\right) d y d x
$$

as desired, and this concludes the proof of the result.

\section{Monotonicity results for the period function}

Proposition 3.1. Suppose that there exist $\eta_{1}, \eta_{2} \in \mathbb{R}$ with $\eta_{1}+\eta_{2}=1$ such that, for $i=1,2, \mathscr{P}_{\sigma_{i}}\left(\ell_{i}^{\prime}(z)-\eta_{i}\right)$ is positive (respectively, negative) for all $z \in\left(0, e_{i}^{+}\right)$. Then the period function of the center at the origin of the differential system (1) is monotonous increasing (respectively, decreasing).

Proof. By applying Lemma 2.3 and $(a)$ in Proposition 2.7 we have that

$$
h T^{\prime}(h)=4 \int_{Q_{1} \cap \gamma_{h}}\left(\mathscr{P}_{\sigma_{1}}\left(\ell_{1}^{\prime}-\eta_{1}\right)(x) \mathscr{P}_{\sigma_{2}}(1)(y)+\mathscr{P}_{\sigma_{1}}(1)(x) \mathscr{P}_{\sigma_{2}}\left(\ell_{2}^{\prime}-\eta_{2}\right)(y)\right) \frac{d x}{F_{2}^{\prime}(y)},
$$

where recall that $Q_{1}$ stands for the first quadrant. Since $Q_{1} \cap \gamma_{h} \subset\left(0, e_{1}^{+}\right) \times\left(0, e_{2}^{+}\right)$and, on the other hand, $\mathscr{P}_{\sigma_{i}}(1)(z)=1-\sigma_{i}^{\prime}(z)>0$, the result follows.

Next result is addressed to non-degenerate centers and it is in fact a consequence of Proposition 3.1 because it provides a sufficient condition in order that $\mathscr{P}_{\sigma_{i}}\left(\ell_{i}^{\prime}-\frac{1}{2}\right)$ does not vanish.

Proposition 3.2. Assume that the center at the origin is non-degenerate, i.e., $k_{1}=k_{2}=2$. If the function $\mathscr{P}_{\sigma_{i}}\left(\left(\frac{F_{i}}{\left(F_{i}^{\prime}\right)^{2}}\right)^{\prime \prime}\right)$ is positive (respectively, negative) on $\left(0, e_{i}^{+}\right)$for $i=1,2$, then the period function of the center at the origin of the differential system (1) is monotonous increasing (respectively, decreasing).

Proof. The result will follow by applying Proposition 3.1 with $\eta_{1}=\eta_{2}=\frac{1}{2}$. With this aim in view note first that, due to $F_{i}^{\prime}(z)=F_{i}^{\prime}\left(\sigma_{i}(z)\right) \sigma_{i}^{\prime}(z)$,

$$
\mathscr{P}_{\sigma_{i}}\left(\ell_{i}^{\prime}-\frac{1}{2}\right)(z)=\left(\ell_{i}^{\prime}-\frac{1}{2}\right)(z)-\left(\ell_{i}^{\prime}-\frac{1}{2}\right)\left(\sigma_{i}(z)\right) \sigma_{i}^{\prime}(z)=F_{i}^{\prime}(z)\left(\left(\frac{\ell_{i}^{\prime}-\frac{1}{2}}{F_{i}^{\prime}}\right)(z)-\left(\frac{\ell_{i}^{\prime}-\frac{1}{2}}{F_{i}^{\prime}}\right)\left(\sigma_{i}(z)\right)\right) .
$$

Observe in addition that, since $F_{i}(z)=\alpha_{i} z^{2}+\mathrm{o}\left(z^{2}\right)$, we have $\ell_{i}(z)=\frac{1}{2} z+\mathrm{o}(z)$. Therefore $\left(\frac{\ell_{i}^{\prime}-\frac{1}{2}}{F_{i}^{\prime}}\right)(z)$ is an analytic function at $z=0$ and

$$
\left(\frac{\ell_{i}^{\prime}-\frac{1}{2}}{F_{i}^{\prime}}\right)(z)-\left.\left(\frac{\ell_{i}^{\prime}-\frac{1}{2}}{F_{i}^{\prime}}\right)\left(\sigma_{i}(z)\right)\right|_{z=0}=0 .
$$

Consequently, from (3) and on account of $F_{i}^{\prime}(z)>0$ for all $z \in\left(0, e_{i}^{+}\right)$, a sufficient condition for $\mathscr{P}_{\sigma_{i}}\left(\ell_{i}^{\prime}-\frac{1}{2}\right)$ to be positive (respectively, negative) on $\left(0, e_{i}^{+}\right)$is that

$$
\frac{d}{d z}\left(\left(\frac{\ell_{i}^{\prime}-\frac{1}{2}}{F_{i}^{\prime}}\right)(z)-\left(\frac{\ell_{i}^{\prime}-\frac{1}{2}}{F_{i}^{\prime}}\right)\left(\sigma_{i}(z)\right)\right)=\mathscr{P}_{\sigma_{i}}\left(\left(\frac{\ell_{i}^{\prime}-\frac{1}{2}}{F_{i}^{\prime}}\right)^{\prime}\right)(z)
$$

is positive (respectively, negative) for $z \in\left(0, e_{i}^{+}\right)$. This proves the result because $\frac{\ell_{i}^{\prime}-\frac{1}{2}}{F_{i}^{\prime}}=\frac{1}{2}\left(\frac{F_{i}}{\left(F_{i}^{\prime}\right)^{2}}\right)^{\prime}$. 
In general one cannot expect to have the explicit expression of the involutions $\sigma_{i}$ and this certainly diminishes the applicability of Propositions 3.1 and 3.2. There are however situations where we can bypass this obstruction and study effectively $\mathscr{P}_{\sigma_{i}}\left(\ell_{i}^{\prime}-\eta_{i}\right)$ without knowing explicitly $\sigma_{i}$. This can be done for instance in case that $F_{i}$ are algebraic functions with the aid of the multipolynomial resultant (see [10, 11, 20, 21] for examples of application of this approach). Alternatively one can seek (explicit) conditions on $F_{i}$ implying that $\mathscr{P}_{\sigma_{i}}\left(\ell_{i}^{\prime}-\eta_{i}\right)$ is non-vanishing. This is in fact the underlying idea in the monotonicity criterion obtained by Schaaf [31]. More concretely he showed that if the conditions

$$
5 F_{i}^{\prime \prime \prime}(z)^{2}-3 F_{i}^{\prime \prime}(z) F_{i}^{(4)}(z)>0 \text { for any } z \in\left(e_{i}^{-}, e_{i}^{+}\right) \text {with } F_{i}^{\prime \prime}(z)>0
$$

and

$$
F_{i}^{\prime}(z) F_{i}^{\prime \prime \prime}(z)<0 \text { for any } z \in\left(e_{i}^{-}, e_{i}^{+}\right) \text {with } F_{i}^{\prime \prime}(z)=0
$$

hold then $\mathscr{P}_{\sigma_{i}}\left(\ell_{i}^{\prime}(z)-\frac{1}{2}\right)>0$ for all $z \in\left(0, e_{i}^{+}\right)$. Likewise Schaaf also proved that if

$$
5 F_{i}^{\prime \prime \prime}(z)^{2}-3 F_{i}^{\prime \prime}(z) F_{i}^{(4)}(z)<0 \text { for any } z \in\left(e_{i}^{-}, e_{i}^{+}\right) \text {with } F_{i}^{\prime \prime}(z) \geqslant 0
$$

then $\mathscr{P}_{\sigma_{i}}\left(\ell_{i}^{\prime}(z)-\frac{1}{2}\right)<0$ for all $z \in\left(0, e_{i}^{+}\right)$. Interestingly enough, as the author points out, the first sufficient condition is related to the Schwarzian derivative. Later on Rothe [25] extended and studied systematically this type of sufficient conditions. Next we shall obtain two of these sufficient conditions in Corollaries 3.4 and 3.5. Before that we prove the following technical result.

Lemma 3.3. (a) Let $a_{1}, a_{2}, \ldots, a_{n}$ and $b_{1}, b_{2}, \ldots, b_{n}$ be analytic functions on $\left(0, e_{1}^{+}\right)$and $\left(0, e_{2}^{+}\right)$, respectively, and consider the annulus

$$
\mathscr{A}=\bigcup_{h \in\left(h_{1}, h_{2}\right)} \gamma_{h} \text { with } 0 \leqslant h_{1}<h_{2} \leqslant h_{0}
$$

Then $\sum_{i=1}^{n} \mathscr{P}_{\sigma_{1}}\left(a_{i}\right)(x) \mathscr{P}_{\sigma_{2}}\left(b_{i}\right)(y)$ is positive (respectively, negative) on $Q_{1} \cap \mathscr{A}$ if $\sum_{i=1}^{n} a_{i}(x) b_{i}(y)$ is positive (respectively, negative) on $\mathscr{A}$.

(b) $\mathscr{P}_{\sigma_{i}}(f)$ is positive (respectively, negative) on $\left(0, e_{i}^{+}\right)$if $f$ is a positive (respectively, negative) function on $\left(e_{i}^{-}, e_{i}^{+}\right) \backslash\{0\}$.

(c) $\mathscr{P}_{\sigma_{i}}(f)$ is positive (respectively, negative) on $\left(0, e_{i}^{+}\right)$if $\frac{R\left(F_{i}\right) f}{F_{i}^{\prime}}$ is a smooth monotonous increasing (respectively, decreasing) function on $\left(e_{i}^{-}, e_{i}^{+}\right)$, where $R$ is any function with $R(h)>0$ for all $h \in\left(0, h_{0}\right)$.

Proof. To show $(a)$ assume for instance that $\sum_{i=1}^{n} a_{i}(x) b_{i}(y)>0$ for all $(x, y) \in \mathscr{A}$. Let $\mathscr{A}_{i}$ denote the intersection of $\mathscr{A}$ with the $i$-th quadrant. Recall that $\sigma_{i}^{\prime}<0$ since $F_{i}^{\prime}\left(\sigma_{i}(z)\right) \sigma_{i}^{\prime}(z)=F_{i}^{\prime}(z)$ for all $z \in\left(e_{i}^{-}, e_{i}^{+}\right)$. Thus, if $(x, y) \in \mathscr{A}_{1} \cup \mathscr{A}_{2}$ then $-\sigma_{2}^{\prime}(y) \sum_{i=1}^{n} a_{i}(x) b_{i}\left(\sigma_{2}(y)\right)>0$ because $\left(x, \sigma_{2}(y)\right) \in \mathscr{A}_{3} \cup \mathscr{A}_{4} \subset \mathscr{A}_{\text {. Hence, }}$ by definition,

$$
2 \sum_{i=1}^{n} a_{i}(x) \mathscr{P}_{\sigma_{2}}\left(b_{i}\right)(y)=\sum_{i=1}^{n}\left(a_{i}(x) b_{i}(y)-a_{i}(x) b_{i}\left(\sigma_{2}(y)\right) \sigma_{2}^{\prime}(y)\right)>0 \text { for all }(x, y) \in \mathscr{A}_{1} \cup \mathscr{A}_{2} \text {. }
$$

On account of this, if $(x, y) \in \mathscr{A}_{1}$ then, since $\left(\sigma_{1}(x), y\right) \in \mathscr{A}_{2}$, we get $-\sigma_{1}^{\prime}(x) \sum_{i=1}^{n} a_{i}\left(\sigma_{1}(x)\right) \mathscr{P}_{\sigma_{2}}\left(b_{i}\right)(y)>0$, which by definition implies

$$
2 \sum_{i=1}^{n} \mathscr{P}_{\sigma_{1}}\left(a_{i}\right)(x) \mathscr{P}_{\sigma_{2}}\left(b_{i}\right)(y)=\sum_{i=1}^{n}\left(a_{i}(x) \mathscr{P}_{\sigma_{2}}\left(b_{i}\right)(y)-a_{i}\left(\sigma_{1}(x)\right) \mathscr{P}_{\sigma_{2}}\left(b_{i}\right)(y) \sigma_{1}^{\prime}(x)\right)>0 .
$$


This proves the validity of $(a)$. The assertion $(b)$ is obvious from the definition and the fact that $\sigma_{i}^{\prime}<0$. Finally in order to show $(c)$ note that, on account of $F_{i}(z)=F_{i}\left(\sigma_{i}(z)\right)$ for all $z$,

$$
2 \mathscr{P}_{\sigma_{i}}(f)(z)=f(z)-f\left(\sigma_{i}(z)\right) \frac{F_{i}^{\prime}(z)}{F_{i}^{\prime}\left(\sigma_{i}(z)\right)}=\left(\frac{F_{i}^{\prime}}{R\left(F_{i}\right)}\right)(z)\left(\left(\frac{R\left(F_{i}\right) f}{F_{i}^{\prime}}\right)(z)-\left(\frac{R\left(F_{i}\right) f}{F_{i}^{\prime}}\right)\left(\sigma_{i}(z)\right)\right) .
$$

Since $\sigma_{i}(z)<0<z$ for all $z \in\left(0, e_{i}^{+}\right)$and $F_{i}\left(\left(0, e_{i}^{+}\right)\right)=\left(0, h_{0}\right)$, the assertion in $(c)$ follows thanks to $\left(\frac{F_{i}^{\prime}}{R\left(F_{i}\right)}\right)(z)>0$ for all $z \in\left(0, e_{i}^{+}\right)$and the monotonicity assumption of $\frac{R\left(F_{i}\right) f}{F_{i}^{\prime}}$. This proves the result.

The following result is a consequence of Proposition 3.1 and $(b)$ in Lemma 3.3. For non-degenerate centers it was previously obtained by Rothe [25, Theorem 1], see also [9, Proposition 10].

Corollary 3.4. Suppose that there exist $\eta_{1}, \eta_{2} \in \mathbb{R}$ with $\eta_{1}+\eta_{2}=1$ such that, for $i=1,2, \ell_{i}^{\prime}(z)-\eta_{i}$ is positive (respectively, negative) for all $z \in\left(e_{i}^{-}, e_{i}^{+}\right) \backslash\{0\}$. Then the period function of the center at the origin of the differential system (1) is monotonous increasing (respectively, decreasing).

Next result was proved initially by Chicone [2, Theorem A] for potential systems $H(x, y)=\frac{1}{2} y^{2}+V(x)$ and it was later extended in $[9,25]$ for general systems $H(x, y)=F_{1}(x)+F_{2}(y)$. It follows by applying Proposition 3.2 and taking account of $(b)$ in Lemma 3.3.

Corollary 3.5. Assume that the center at the origin is non-degenerate, i.e., $k_{1}=k_{2}=2$. If $\left(\frac{F_{i}}{\left(F_{i}^{\prime}\right)^{2}}\right)^{\prime \prime}$ is positive (respectively, negative) on $\left(e_{i}^{-}, e_{i}^{+}\right) \backslash\{0\}$ for $i=1,2$, then the period function of the center at the origin of the differential system (1) is monotonous increasing (respectively, decreasing).

A computation shows that

$$
\left(\frac{F}{\left(F^{\prime}\right)^{2}}\right)^{\prime}=\frac{\left(F^{\prime}\right)^{2}-2 F F^{\prime \prime}}{\left(F^{\prime}\right)^{3}} \text { and }\left(\frac{F}{\left(F^{\prime}\right)^{2}}\right)^{\prime \prime}=\frac{2 F F^{\prime} F^{\prime \prime \prime}-6 F\left(F^{\prime \prime}\right)^{2}-3\left(F^{\prime}\right)^{2} F^{\prime \prime}}{\left(F^{\prime}\right)^{4}}
$$

The following result is valid for degenerate centers as well and it constitutes our last application of Proposition 3.2. In this case we will use $(c)$ in Lemma 3.3 taking $R(z)=z$. Let us remark that other choices for $R$ will lead to new monotonicity criteria, certainly with longer expressions but perhaps more convenient in order to study specific systems.

Corollary 3.6. Suppose that there exist $\eta_{1}, \eta_{2} \in \mathbb{R}$ with $\eta_{1}+\eta_{2}=1$ such that $\ell_{i}\left(\ell_{i}^{\prime}-\eta_{i}\right)$ is monotonous increasing (respectively, decreasing) on $\left(e_{i}^{-}, e_{i}^{+}\right)$for $i=1,2$. Then the period function of the center at the origin of the differential system (1) is monotonous increasing (respectively, decreasing).

Proof. By Proposition 3.1 it suffices to show that, for $i=1,2, \mathscr{P}_{\sigma_{i}}\left(\ell_{i}^{\prime}(z)-\eta_{i}\right)$ is positive (respectively, negative) for all $z \in\left(0, e_{i}^{+}\right)$. In turn, by applying $(c)$ in Lemma 3.3 with $R(z)=z$, a sufficient condition for this to hold is that $\frac{F_{i}}{F_{i}^{\prime}}\left(\ell_{i}^{\prime}-\eta_{i}\right)$ is monotonous increasing (respectively, decreasing) on $\left(e_{i}^{-}, e_{i}^{+}\right)$.

The monotonicity criteria that we obtained so far require that two functions of a single variable do not vanish in an interval. This provides sufficient conditions for monotonicity that are easy to verify. The disadvantage is that these conditions are perhaps excessively far away from being necessary. In what follows we will try to amend this by giving sufficient conditions that concern two-variable functions. The following is the first one of the results in this direction.

Proposition 3.7. The period function of the center at the origin of the differential system (1) verifies

$$
h^{2} T^{\prime}(h)=4 \iint_{Q_{1} \cap \operatorname{Int}\left(\gamma_{h}\right)} K(x, y) d y d x
$$


where

$$
\begin{aligned}
K(x, y):=2 \mathscr{P}_{\sigma_{1}}\left(\ell_{1}^{\prime}\right)(x) \mathscr{P}_{\sigma_{2}}\left(\ell_{2}^{\prime}\right)(y) & +\mathscr{P}_{\sigma_{1}}\left(\left(\ell_{1}\left(\ell_{1}^{\prime}-1\right)\right)^{\prime}\right)(x) \mathscr{P}_{\sigma_{2}}(1)(y) \\
& +\mathscr{P}_{\sigma_{2}}\left(\left(\ell_{2}\left(\ell_{2}^{\prime}-1\right)\right)^{\prime}\right)(y) \mathscr{P}_{\sigma_{1}}(1)(x) .
\end{aligned}
$$

Moreover there are no critical periodic orbits in the period annulus if $K$ does not change sign on $Q_{1} \cap \mathscr{P}$. Finally, a sufficient condition for the latter to hold is that

$$
\hat{K}(x, y):=2 \ell_{1}^{\prime}(x) \ell_{2}^{\prime}(y)+\left(\ell_{1}\left(\ell_{1}^{\prime}-1\right)\right)^{\prime}(x)+\left(\ell_{2}\left(\ell_{2}^{\prime}-1\right)\right)^{\prime}(y)
$$

does not change sign on $\mathscr{P}$.

Proof. By Lemma 2.3 we can assert that

$$
h T^{\prime}(h)=\int_{\gamma_{h}}\left(\ell_{1}^{\prime}(x)+\ell_{2}^{\prime}(y)-1\right) \frac{d x}{F_{2}^{\prime}(y)} .
$$

Then the expression for $h^{2} T^{\prime}(h)$ follows by applying $(b)$ in Proposition 2.7 three times, with $\left\{a=\ell_{1}^{\prime}, b=1\right\}$, $\left\{a=1, b=\ell_{2}^{\prime}\right\}$ and $\{a=b=1\}$, and performing afterwards some easy simplifications. From this expression it is clear that a sufficient condition for $T^{\prime}(h) \neq 0$ for all $h \in\left(0, h_{0}\right)$ is that $K$ does not change sign on $Q_{1} \cap \mathscr{P}$. In its turn, by $(a)$ in Lemma 3.3, a sufficient condition for this to be verified is that $\hat{K}$ does not change sign on $\mathscr{P}$. This proves the result.

Remark 3.8. For reader's convenience let us note that

$$
\hat{K}(x, y)=2\left(\frac{F_{1}}{F_{1}^{\prime}}\right)^{\prime}(x)\left(\frac{F_{2}}{F_{2}^{\prime}}\right)^{\prime}(y)-\left(\frac{F_{1}^{2} F_{1}^{\prime \prime}}{\left(F_{1}^{\prime}\right)^{3}}\right)^{\prime}(x)-\left(\frac{F_{2}^{2} F_{2}^{\prime \prime}}{\left(F_{2}^{\prime}\right)^{3}}\right)^{\prime}(y) .
$$

On the other hand, with regard to the non-vanishing assumption of the function $K$ in Proposition 3.7, we note that if $F_{i}(z)=\alpha_{i} z^{k_{i}}+\mathrm{o}\left(z^{k_{i}}\right)$ then one can check that $K(0,0)=\eta(\eta-1)$, with $\eta:=\frac{1}{k_{1}}+\frac{1}{k_{2}}$. Hence $K(0,0)$ is negative if the center is degenerate (i.e., when either $k_{1}>2$ or $k_{2}>2$ ) and zero otherwise (i.e., when $k_{1}=k_{2}=2$ ). Thus, in the first case the period function is monotonous decreasing for $h \approx 0$, which is consistent with the well known fact that, for degenerate centers, $T(h) \longrightarrow+\infty$ as $h$ tends to zero.

\section{Criteria for at most one critical periodic orbit}

In the statement of our next result $\mathscr{A}$ stands for an open annulus in $\mathscr{P}$ as introduced in (4). By taking $\mathscr{A}$ to be the whole $\mathscr{P}$ we obtain a criterion for the existence of at most one critical periodic orbit.

Theorem 4.1. If there exist $\alpha, \beta \in \mathbb{R}$ with $\alpha+\beta>1$ (respectively, $\alpha+\beta<1$ ) such that the function

$$
\begin{aligned}
Q(x, y):=2 \mathscr{P}_{\sigma_{1}}\left(\ell_{1}^{\prime}\right)(x) \mathscr{P}_{\sigma_{2}}\left(\ell_{2}^{\prime}\right)(y) & +\mathscr{P}_{\sigma_{1}}\left(\left(\ell_{1}\left(\ell_{1}^{\prime}-\alpha\right)\right)^{\prime}\right)(x) \mathscr{P}_{\sigma_{2}}(1)(y) \\
& +\mathscr{P}_{\sigma_{2}}\left(\left(\ell_{2}\left(\ell_{2}^{\prime}-\alpha\right)\right)^{\prime}\right)(y) \mathscr{P}_{\sigma_{1}}(1)(x)-\beta \mathscr{P}_{\sigma_{1}}(1)(x) \mathscr{P}_{\sigma_{2}}(1)(y)
\end{aligned}
$$

is positive (respectively, negative) on $Q_{1} \cap \mathscr{A}$, then there exists at most one critical periodic orbit inside $\mathscr{A}$, multiplicities taking into account, and it is a minimum (respectively, maximum). In addition, a sufficient condition for this to hold is that

$$
\hat{Q}(x, y):=2 \ell_{1}^{\prime}(x) \ell_{2}^{\prime}(y)+\left(\ell_{1}\left(\ell_{1}^{\prime}-\alpha\right)\right)^{\prime}(x)+\left(\ell_{2}\left(\ell_{2}^{\prime}-\alpha\right)\right)^{\prime}(y)-\beta
$$

is positive (respectively, negative) on $\mathscr{A}$. 
Proof. Let us consider $A(h):=\int_{\gamma_{h}} y d x$ for $h \in\left(0, h_{0}\right)$. Clearly $A(h)$ is the area of the $\operatorname{region} \operatorname{Int}\left(\gamma_{h}\right)$ and a general well known result on the period function of Hamiltonian differential systems asserts that $A^{\prime}(h)=T(h)$, see for instance [19, Theorem A]. As a matter of fact, for the particular Hamiltonians that we deal with, this can be shown by applying Lemma 2.2 because in doing so we get

$$
A^{\prime}(h)=\frac{d}{d h} \int_{\gamma_{h}} y d x=\int_{\gamma_{h}} \frac{d x}{F_{2}^{\prime}(y)}=T(h) .
$$

Note in addition that, by applying Green's Theorem and taking $F_{i} \circ \sigma_{i}=F_{i}$ for $i=1,2$ into account,

$$
A(h)=\iint_{\operatorname{Int}\left(\gamma_{h}\right)} d y d x=4 \iint_{Q_{1} \cap \operatorname{Int}\left(\gamma_{h}\right)} \mathscr{P}_{\sigma_{1}}(1)(x) \mathscr{P}_{\sigma_{2}}(1)(y) d y d x .
$$

On the other hand, by applying $(b)$ in Proposition 2.7 with $\{a=b=1\}$ we get

$$
h T(h)=4 \iint_{Q_{1} \cap \operatorname{Int}\left(\gamma_{h}\right)}\left(\mathscr{P}_{\sigma_{1}}\left(\ell_{1}^{\prime}\right)(x) \mathscr{P}_{\sigma_{2}}(1)(y)+\mathscr{P}_{\sigma_{1}}(1)(x) \mathscr{P}_{\sigma_{2}}\left(\ell_{2}^{\prime}\right)(x)\right) d y d x .
$$

The two previous identities, together with the one given in Proposition 3.7, yield

$$
G(h):=h^{2} T^{\prime}(h)+(1-\alpha) h T(h)-\beta A(h)=4 \iint_{Q_{1} \cap \operatorname{Int}\left(\gamma_{h}\right)} Q(x, y) d y d x
$$

because one can verify that

$$
Q(x, y)=K(x, y)+(1-\alpha)\left(\mathscr{P}_{\sigma_{1}}\left(\ell_{1}^{\prime}\right)(x) \mathscr{P}_{\sigma_{2}}(1)(y)+\mathscr{P}_{\sigma_{1}}(1)(x) \mathscr{P}_{\sigma_{2}}\left(\ell_{2}^{\prime}\right)(x)\right)-\beta \mathscr{P}_{\sigma_{1}}(1)(x) \mathscr{P}_{\sigma_{2}}(1)(y) .
$$

We are now in position to prove the result. To this end assume $\alpha+\beta>1$ and that $Q$ is positive on $Q_{1} \cap \mathscr{A}$ (the other case follows exactly the same way). Then for any $h, \hat{h} \in\left(h_{1}, h_{2}\right)$ with $h<\hat{h}$ we have

$$
G(\hat{h})-G(h)=\iint_{Q_{1} \cap\left(\operatorname{Int}\left(\gamma_{\hat{h}}\right) \backslash \operatorname{Int}\left(\gamma_{h}\right)\right)} Q(x, y) d y d x>0 .
$$

Accordingly $G$ is a monotonous increasing function on $\left(h_{1}, h_{2}\right)$. Therefore, on account of $A^{\prime}=T$,

$$
G^{\prime}(h)=h^{2} T^{\prime \prime}(h)+(2+1-\alpha) h T^{\prime}(h)+(1-\alpha-\beta) T(h) \geqslant 0 \text { for all } h \in\left(h_{1}, h_{2}\right) .
$$

If $\gamma_{h_{\star}}$ is a critical periodic orbit in $\mathscr{A}$, i.e, $T^{\prime}\left(h_{\star}\right)=0$, then $h_{\star}^{2} T^{\prime \prime}\left(h_{\star}\right)+(1-\alpha-\beta) T\left(h_{\star}\right) \geqslant 0$, which implies $h_{\star}^{2} T^{\prime \prime}\left(h_{\star}\right) \geqslant(\alpha+\beta-1) T\left(h_{\star}\right)>0$ thanks to the hypothesis $\alpha+\beta>1$. This shows, simultaneously, that the critical periodic orbit must be a minimum and that there exists at most one critical periodic orbit in $\mathscr{A}$, multiplicities taking into account. This proves the first assertion in the statement whereas the second one follows by $(a)$ in Lemma 3.3. So the result is proved.

Remark 4.2. The sign of $Q$ near the origin is relevant in case that Theorem 4.1 is applied with $\mathscr{A}=\mathscr{P}$. In this respect, since $F_{i}(x)=a_{i} x^{k_{i}}+\mathrm{o}\left(z^{k_{i}}\right)$ with $k_{i}$ an even number, note that $Q(0,0)=\eta^{2}-\alpha \eta-\beta$, where $\eta=\frac{1}{k_{1}}+\frac{1}{k_{2}} \in(0,1]$.

Remark 4.3. At this point it is to be referred Sabatini's paper [30], where to the best of our knowledge it is given the only criterion that appears in the literature to ensure the existence of at most one critical periodic orbit for Hamiltonian systems with separable variables. In short, the author introduces a function $\mu_{s 2}=\mu_{s 2}(x, y)$ and shows that the Hamiltonian system has at most one critical periodic orbit if $\mu_{s 2}$ has constant sign on $\mathscr{P}$. His result is in some way complementary to Theorem 4.1 because one can verify that $\mu_{s 2}$ is the function $\hat{Q}$ taking $\alpha=2$ and $\beta=-1$. The approach in that paper is completely different to the one we follow here and it relies in the use of the so-called normalizers. 
In this setting there is another paper by Sabatini that is worth to mention. Indeed he proved a result, see [29, Theorem 1], that provides sufficient conditions for the existence of at most one critical periodic orbit in an annulus $\mathscr{A}$ inside $\mathscr{P}$. However, when applied to the case $\mathscr{A}=\mathscr{P}$, conditions $L_{2}$ and $L_{3}$ in that result imply the monotonicity of the period function. (This last assertion can be shown, for instance, by applying Corollary 3.4.) It occurs the same with Rothe's result [25, Theorem 3], which provides a criterion for $T^{\prime}(h)>0$ and $\left(\frac{h T^{\prime}(h)}{T(h)}\right)^{\prime}>0$ for all $h \in\left(0, h_{0}\right)$. The second inequality easily implies the existence of at most one critical periodic orbit in $\mathscr{P}$ but certainly it does not constitute a uniqueness criterion for (non-monotonous) period functions.

To the best of our knowledge, in the literature there are no examples apart from the potential systems of Hamiltonian centers with $H(x, y)=F_{1}(x)+F_{2}(y)$ for which it has been established the existence of exactly one critical periodic orbit. Next we give two examples of this by applying Theorem 4.1. Let us remark that we tried to avoid technicalities due to computational issues and so although it would be possible to tackle examples with $F_{i}$ not being even functions or having parameters, we prefer not to do it.

Example 4.4. We begin by studying the degenerate center given by $H(x, y)=F_{1}(x)+F_{2}(y)$ with

$$
F_{1}(z)=\frac{1}{4} z^{4}-\frac{1}{8} z^{8} \text { and } F_{2}(z)=\frac{1}{6} z^{6}-\frac{1}{10} z^{10}
$$

It is easy to verify that $F_{i}^{\prime}(z)=0$ if and only if $z=0$ or $z= \pm 1$. Consequently the period annulus is bounded and, due $F_{1}(1)>F_{2}(1)$, its outer boundary is given by $\left\{F_{1}(x)+F_{2}(y)=F_{2}(1)\right\}$, which is a polycycle that consists in two hyperbolic saddles located at $(0, \pm 1)$ together with two trajectories connecting them. Hence $\lim _{h \rightarrow h_{0}^{-}} T(h)=+\infty$. Since on the other hand we have $\lim _{h \rightarrow 0^{+}} T(h)=+\infty$ due to the fact that the center is degenerate, we can already assert the existence of at least one critical periodic orbit. By applying Theorem 4.1 we shall prove that there exists exactly one. Since $F_{i}$ is an even function for $i=1,2$, note that $\sigma_{i}=-I d$ and $\ell_{i}=\frac{F_{i}}{F_{i}^{\prime}}$ is odd. Then it turns out that

$$
Q(x, y)=2 \ell_{1}^{\prime}(x) \ell_{2}^{\prime}(y)+\left(\ell_{1}\left(\ell_{1}^{\prime}-\alpha\right)\right)^{\prime}(x)+\left(\ell_{2}\left(\ell_{2}^{\prime}-\alpha\right)\right)^{\prime}(y)-\beta .
$$

Taking $\alpha=5 / 2$ and $\beta=-1$, one can verify that

$$
\begin{aligned}
Q(x, y)= & 1900-13600 x^{4}+50925 x^{8}-20592 y^{12}+5125 x^{16}+3564 y^{16}-33550 x^{12}-12400 y^{4}+30600 y^{8} \\
& -171600 x^{4} y^{8}+449790 x^{8} y^{8}-296580 x^{12} y^{8}+52590 x^{16} y^{8}+79600 x^{4} y^{4}-245700 x^{8} y^{4} \\
& +114768 x^{4} y^{12}-21456 x^{4} y^{16}-300132 x^{8} y^{12}+63549 x^{8} y^{16}+161800 x^{12} y^{4}+197928 x^{12} y^{12} \\
& -41886 x^{12} y^{16}-26500 x^{16} y^{4}-35172 x^{16} y^{12}+7029 x^{16} y^{16}
\end{aligned}
$$

and hence the problem reduces to check that this two variable polynomial is positive on $\mathscr{P}$. This can be proved analytically with the help of an algebraic manipulator in several different ways. One possibility is to show that if $p_{0} \in \mathscr{P}$ is a critical point of $Q$, i.e., $\partial_{x} Q\left(p_{0}\right)=\partial_{y} Q\left(p_{0}\right)=0$, then $Q\left(p_{0}\right)>0$ and that, on the other hand, $Q(p)>0$ for all $p \in \partial \mathscr{P}$. Let us explain this skiping the computational details for the sake of shortness. The possible critical points can be isolated in arbitrarily small boxes by computing the two resultants between $\partial_{x} Q$ and $\partial_{y} Q$. In doing so we get twelve boxes inside the rectangle $[0,0.76] \times[0,1]$, which certainly contains $Q_{1} \cap \mathscr{P}$ because the smallest positive root of $F_{1}(x)=F_{2}(1)$ is $x \approx 0.7502$. Then we prove, also analytically, that $Q$ has a positive lower bound in each one of these twelve small boxes. Finally to show that $Q(p)>0$ for all $p \in \partial \mathscr{P}$ we compute the resultant with respect to $x$ between $Q(x, y)$ and $F_{1}(x)+F_{2}(y)-F_{2}(1)$ and we verify next by Sturm's Theorem that the polynomial in $y$ that thus obtain does not vanish on $(0,1)$.

Example 4.5. Let us now study $H(x, y)=F_{1}(x)+F_{2}(y)$ with

$$
F_{1}(z)=\frac{z^{4}}{1+2 z^{6}} \text { and } F_{2}(z)=\frac{z^{4}}{1+z^{8}} \text {. }
$$


One can check that $F_{i}^{\prime}(z)=0$ if and only if $z=0$ or $z= \pm 1$. In this case $F_{1}(1)<F_{2}(1)$ and so the outer boundary of $\mathscr{P}$ is $\left\{F_{1}(x)+F_{2}(y)=F_{1}(1)\right\}$. Moreover, since the smallest positive root of $F_{2}(y)=F_{1}(1)$ is $y \approx 0.7861$, we can assert that $Q_{1} \cap \mathscr{P}$ is inside the rectangle [0,1] $\times[0.79]$. Exactly as in the previous example, there exists at least one critical periodic orbit because the center is bounded and degenerate. We apply Theorem 4.1 taking $\alpha=9 / 4$ and $\beta=-1$ to obtain $Q(x, y)=\frac{S(x, y)}{16\left(x^{6}-1\right)^{4}\left(y^{8}-1\right)^{4}}$ with

$$
\begin{aligned}
S(x, y)= & +13 x^{6}+16 y^{32}-78 y^{24}-89 x^{18}+26 x^{24}+782 y^{16}+1020 x^{12}+488 x^{6} y^{8}-4598 x^{6} y^{16} \\
& -5376 x^{12} y^{8}+14160 x^{12} y^{16}+1112 x^{18} y^{8}-5666 x^{18} y^{16}-158 x^{24} y^{8}+1154 x^{24} y^{16}+1152 x^{6} y^{24} \\
& -127 x^{6} y^{32}-6480 x^{12} y^{24}+1284 x^{12} y^{32}+1824 x^{18} y^{24}-253 x^{18} y^{32}-306 x^{24} y^{24}+52 x^{24} y^{32}+46 y^{8} .
\end{aligned}
$$

As before the problem reduces to show that this polynomial is positive in the rectangle $[0,1] \times[0.79]$ but in this case we argue differently. We collect it as $Q(x, y)=p_{0}(y)+p_{1}(y) x^{6}+p_{2}(y) x^{12}+p_{3}(y) x^{18}+p_{4}(y) x^{24}$ and we show that the lower bounds

$$
p_{0}(y)>2, p_{1}(y)>12, p_{2}(y)>486, p_{3}(y)>-90 \text { and } p_{4}(y)>20
$$

hold for all $y \in[0,0.79]$. Thus $Q(x, y)>2+12 x^{6}+486 x^{12}-90 x^{18}+20 x^{24}$ for all $(x, y) \in \mathscr{P}$ and, on the other hand, by applying Sturm's Theorem one can prove that the polynomial in $x$ is positive on $(0,1)$.

Proposition 3.2 shows that if the center is non-degenerate and, for $i=1,2$, the function $\mathscr{P}_{\sigma_{i}}\left(\left(\frac{F_{i}}{\left(F_{i}^{\prime}\right)^{2}}\right)^{\prime \prime}\right)$ does not vanish on $\left(0, e_{i}^{+}\right)$then there are no critical periodic orbits. Next we will show that, under some additional hypothesis, if this function has exactly one zero on $\left(0, e_{i}^{+}\right)$then the center has at most one critical periodic orbit. To see this we shall appeal to some tools developed in $[11,20]$ and to this end some definitions are needed. The first one is the following, see for instance [14].

Definition 4.6. Let $f_{0}, f_{1}, \ldots, f_{n-1}$ be analytic functions on an open interval $L$ of $\mathbb{R}$.

(a) The ordered set $\left(f_{0}, f_{1}, \ldots, f_{n-1}\right)$ is a complete Chebyshev system (in short, CT-system) on $L$ if, for all $k=1,2, \ldots, n$, any nontrivial linear combination

$$
\alpha_{0} f_{0}(x)+\alpha_{1} f_{1}(x)+\ldots+\alpha_{k-1} f_{k-1}(x)
$$

has at most $k-1$ isolated zeros on $L$.

(b) The ordered set $\left(f_{0}, f_{1}, \ldots, f_{n-1}\right)$ is an extended complete Chebyshev system (in short, ECT-system) on $L$ if, for all $k=1,2, \ldots, n$, any nontrivial linear combination

$$
\alpha_{0} f_{0}(x)+\alpha_{1} f_{1}(x)+\ldots+\alpha_{k-1} f_{k-1}(x)
$$

has at most $k-1$ isolated zeros on $L$ counted with multiplicities.

It is clear from the previous definitions that any ECT-system is in particular a CT-system. The first ones have an easy characterization in terms of Wronskians, as the next well known result shows (see again [14]).

Lemma 4.7. $\left(f_{0}, f_{1}, \ldots, f_{n-1}\right)$ is an ECT-system on $I$ if and only if none of the leading principal minors of its Wronskian

$$
W\left[f_{0}, f_{1}, \ldots, f_{n-1}\right](x)=\left|\begin{array}{ccc}
f_{0}(x) & \cdots & f_{n-1}(x) \\
f_{0}^{\prime}(x) & \cdots & f_{n-1}^{\prime}(x) \\
& \vdots & \\
f_{0}^{(n-1)}(x) & \cdots & f_{n-1}^{(n-1)}(x)
\end{array}\right|
$$

vanishes on $I$. 
The following result is Theorem $\mathrm{A}$ in [11] and in its statement $f_{0}, f_{1}, \ldots, f_{n}-1$ and $g$ are analytic functions. For reader's convenience we adapt the statement to the definitions we use in the present paper.

Theorem 4.8. Let us consider the Abelian integrals

$$
I_{i}(h)=\int_{\gamma_{h}} f_{i}(x) g(y) d x, \quad i=0,1, \ldots, n-1,
$$

where, for each $h \in\left(0, h_{0}\right), \gamma_{h}$ is the oval surrounding the origin inside the level curve $\left\{F_{1}(x)+F_{2}(y)=h\right\}$. For $i=1,2$, let $\sigma_{i}$ be the involution associated to $F_{i}$ and suppose $k_{i}=2$. Finally, setting $g_{0}=g$, let us define $g_{i+1}=\frac{g_{i}^{\prime}}{F_{2}^{\prime}}$. Then $\left(I_{0}, I_{1}, \ldots, I_{n-1}\right)$ is an ECT-system on $\left(0, h_{0}\right)$ if the following hypothesis are satisfied:

(a) $\left(\mathscr{P}_{\sigma_{1}}\left(f_{0}\right), \mathscr{P}_{\sigma_{1}}\left(f_{1}\right), \ldots, \mathscr{P}_{\sigma_{1}}\left(f_{n-1}\right)\right)$ is a CT-system on $\left(0, e_{1}^{+}\right)$,

(b) $\left(\mathscr{P}_{\sigma_{2}}\left(F_{2}^{\prime} g_{0}\right), \mathscr{P}_{\sigma_{2}}\left(F_{2}^{\prime} g_{1}\right), \ldots, \mathscr{P}_{\sigma_{2}}\left(F_{2}^{\prime} g_{n-1}\right)\right)$ is a CT-system on $\left(0, e_{2}^{+}\right)$and $g(y)-g(-y)=\mathrm{o}\left(y^{2(n-2)}\right)$.

Next result (see [20, Proposition 2.2]) is the last ingredient that we need to borrow from the literature and it refers to the notion of $\sigma$-evenness as introduced in Definition 2.5.

Proposition 4.9. Let $\sigma$ be an analytic involution on $L=(a, b)$ with $\sigma(0)=0$ and consider an analytic $\sigma$-even function $f$ on $L$. Then $f$ has at most $n$ zeros on $(0, b)$ taking multiplicities into account if and only if there exist $g_{0}, g_{1}, \ldots, g_{n-1}$ analytic $\sigma$-even functions on $L$ such that $\left(g_{0}, g_{1}, \ldots, g_{n-1}, f\right)$ is an ECT-system on $(0, b)$.

Lemma 4.10. Assume that the center at the origin is non-degenerate, i.e., $k_{1}=k_{2}=2$. Suppose moreover that either $F_{2}(y)=F_{1}(y)$ for all $y \in I_{2}$ or $F_{2}(y)=F_{1}(-y)$ for all $y \in I_{2}$. Then the period function $T(h)$ of the center at the origin of the differential system (1) verifies

$$
h T^{\prime}(h)=\int_{\gamma_{h}}\left(\frac{F_{1}}{\left(F_{1}^{\prime}\right)^{2}}\right)^{\prime \prime}(x) y d x .
$$

Proof. Let us set $a_{i}:=\ell_{i}^{\prime}-\frac{1}{2}$ for $i=1,2$. We claim that

$$
h T^{\prime}(h)=2 \int_{\gamma_{h}} a_{1}(x) \frac{d x}{F_{2}^{\prime}(y)} .
$$

To see this we use that, by Lemma 2.3,

$$
h T^{\prime}(h)=\int_{\gamma_{h}}\left(a_{1}(x)+a_{2}(y)\right) \frac{d x}{F_{2}^{\prime}(y)} .
$$

Let us consider first the case $F_{2}(y)=F_{1}(y)$. Then $a_{2}(y)=a_{1}(y)$ and

$$
\int_{\gamma_{h}} a_{2}(y) \frac{d x}{F_{2}^{\prime}(y)}=\int_{\gamma_{h}} a_{1}(y) \frac{d x}{F_{2}^{\prime}(y)}=-\int_{\gamma_{h}} a_{1}(y) \frac{d y}{F_{1}^{\prime}(x)}=\int_{\gamma_{h}} a_{1}(u) \frac{d u}{F_{1}^{\prime}(v)}=\int_{\gamma_{h}} a_{1}(u) \frac{d u}{F_{2}^{\prime}(v)},
$$

where in the second equality we used that $\frac{d y}{d x}=-\frac{F_{1}^{\prime}(x)}{F_{2}^{\prime}(y)}$ for all $(x, y) \in \gamma_{h}$ and in the third one we make the (orientating reversing) coordinate change $\{u=y, v=x\}$. Hence the claim is true in this case. Suppose now that $F_{2}(y)=F_{1}(-y)$. Then a computation shows that $a_{2}(y)=a_{1}(-y)$. Thus

$$
\int_{\gamma_{h}} a_{2}(y) \frac{d x}{F_{2}^{\prime}(y)}=\int_{\gamma_{h}} a_{1}(-y) \frac{d x}{F_{2}^{\prime}(y)}=-\int_{\gamma_{h}} a_{1}(-y) \frac{d y}{F_{1}^{\prime}(x)}=-\int_{\gamma_{h}} a_{1}(u) \frac{d u}{F_{1}^{\prime}(-v)}=\int_{\gamma_{h}} a_{1}(u) \frac{d u}{F_{2}^{\prime}(v)}
$$


where in the second equality we use that $\frac{d y}{d x}=-\frac{F_{1}^{\prime}(x)}{F_{2}^{\prime}(y)}$ for all $(x, y) \in \gamma_{h}$ and in the third one we make the (orientating reversing) coordinate change $\{u=-y, v=-x\}$. So the claim is true also in this case.

Finally, thanks to the claim and on account of $F_{1}(x)=\frac{1}{2} x^{2}+\mathrm{o}\left(x^{2}\right)$, we can apply Lemma 2.1 to conclude

$$
h T^{\prime}(h)=2 \int_{\gamma_{h}} a_{1}(x) \frac{d x}{F_{2}^{\prime}(y)}=2 \int_{\gamma_{h}}\left(\frac{a_{1}}{F_{1}^{\prime}}\right)^{\prime}(x) y d x,
$$

which completes the proof of the result because an easy computation shows that $\frac{a_{1}}{F_{1}^{\prime}}=\frac{1}{2}\left(\frac{F_{1}}{\left(F_{1}^{\prime}\right)^{2}}\right)^{\prime}$.

We are now in position to prove the following uniqueness result for critical periodic orbits.

Theorem 4.11. Assume that the center at the origin is non-degenerate, i.e., $k_{1}=k_{2}=2$, and that either $F_{2}(y)=F_{1}(y)$ for all $y \in I_{2}$ or $F_{2}(y)=F_{1}(-y)$ for all $y \in I_{2}$. Then the period function of the center at the origin of system (1) has at most one critical period, multiplicities taking into account, if the following conditions are satisfied:

(a) $\mathscr{P}_{\sigma_{1}}\left(\left(\frac{F_{1}}{\left(F_{1}^{\prime}\right)^{2}}\right)^{\prime \prime}\right)$ has at most one zero on $\left(0, e_{1}^{+}\right)$, counted with multiplicities, and

(b) $\left(\mathscr{P}_{\sigma_{1}}(1)(x)\right)^{2}+\mathscr{P}_{\sigma_{1}}\left(\frac{F_{1}^{\prime \prime}}{\left(F_{1}^{\prime}\right)^{2}}\right)(x) \mathscr{P}_{\sigma_{1}}\left(x F_{1}^{\prime}(x)\right) \neq 0$ for all $x \in\left(0, e_{1}^{+}\right)$.

Proof. By applying Lemma 4.10, it is clear that it suffices to study the zeros of

$$
L(h):=h T^{\prime}(h)=\int_{\gamma_{h}} f_{1}(x) y d x \text { for } h \in\left(0, h_{0}\right) \text {, where we set } f_{1}:=\left(\frac{F_{1}}{\left(F_{1}^{\prime}\right)^{2}}\right)^{\prime \prime} .
$$

We claim that the assumptions guarantee the existence of an analytic function $I_{0}$ on $\left(0, h_{0}\right)$ such that $\left(I_{0}, L\right)$ form an ECT-system on $\left(0, h_{0}\right)$. This implies in particular, recall Definition 4.6 , that $L$ (and so the derivative of the period function) has at most one zero on $\left(0, h_{0}\right)$ counting multiplicities. So the result will follow once we prove the claim. To this end we will use first the hypothesis that $\varphi_{1}:=\mathscr{P}_{\sigma_{1}}\left(f_{1}\right)$ has at most one zero on $\left(0, e_{1}^{+}\right)$, counted with multiplicities. Note that, by Lemma $2.6, \varphi_{1}$ is a $\sigma_{1}$-even function. Hence, by applying Proposition 4.9, there exists another analytic $\sigma_{1}$-even function $\varphi_{0}$ such that $\left(\varphi_{0}, \varphi_{1}\right)$ is an ECT-system on $\left(0, e_{1}^{+}\right)$. In addition, by Lemma 2.6 once again, we can write $\varphi_{0}=\mathscr{P}_{\sigma_{1}}\left(f_{0}\right)$ for some analytic function $f_{0}$ on $\left(e_{1}^{-}, e_{1}^{+}\right)$. We choose $I_{0}$ to be

$$
I_{0}(h):=\int_{\gamma_{h}} f_{0}(x) y d x
$$

and accordingly we must verify that $\left(I_{0}, L\right)$ is indeed an ECT-system on $\left(0, h_{0}\right)$. To this end we shall apply Theorem 4.8 with $n=2$ and $g(y)=y$. So far we have showed that $\left(\mathscr{P}_{\sigma_{1}}\left(f_{0}\right), \mathscr{P}_{\sigma_{1}}\left(f_{1}\right)\right)$ is an ECT-system on $\left(0, e_{1}^{+}\right)$. Since any ECT-system is in particular a CT-system, the hypothesis $(a)$ in Theorem 4.8 is fulfilled. Due to $g(y)=y$, the hypothesis $(b)$ requires $\left(\mathscr{P}_{\sigma_{2}}\left(y F_{2}^{\prime}(y)\right), \mathscr{P}_{\sigma_{2}}(1)\right)$ to be a CT-system on $\left(0, e_{2}^{+}\right)$. We will show, by applying Lemma 4.7, that it is an ECT-system. To this end, since

$$
2 \mathscr{P}_{\sigma_{2}}\left(y F_{2}^{\prime}(y)\right)=y F_{2}^{\prime}(y)-\sigma_{2}(y) F_{2}^{\prime}\left(\sigma_{2}(y)\right) \sigma_{2}^{\prime}(y)=F_{2}^{\prime}(y)\left(y-\sigma_{2}(y)\right)>0 \text { for all } y \in\left(0, e_{2}^{+}\right),
$$

we only need to check that the Wronskian $W\left[\mathscr{P}_{\sigma_{2}}\left(y F_{2}^{\prime}(y)\right), \mathscr{P}_{\sigma_{2}}(1)\right]$ does not vanish on the interval $\left(0, e_{2}^{+}\right)$. In this regard, setting

$$
\mathscr{L}_{i}(z):=\left(\mathscr{P}_{\sigma_{i}}(1)(z)\right)^{2}+\mathscr{P}_{\sigma_{i}}\left(\frac{F_{i}^{\prime \prime}}{\left(F_{i}^{\prime}\right)^{2}}\right)(z) \mathscr{P}_{\sigma_{i}}\left(z F_{i}^{\prime}(z)\right) \text { for } i=1,2
$$

a computation shows that the Wronskian writes as

$$
W\left[\mathscr{P}_{\sigma_{2}}\left(y F_{2}^{\prime}(y)\right), \mathscr{P}_{\sigma_{2}}(1)\right]=-F_{2}^{\prime}(y) \mathscr{L}_{2}(y) .
$$


Recall at this point that by hypothesis either $F_{2}(y)=F_{1}(y)$ or $F_{2}(y)=F_{1}(-y)$, which imply $\sigma_{2}(y)=\sigma_{1}(y)$ and $\sigma_{2}(y)=-\sigma_{1}(-y)$, respectively. Therefore $\mathscr{L}_{2}(y)=\mathscr{L}_{1}(y)$ for all $y \in I_{2}$ in the first case, whereas one can check that $\mathscr{L}_{2}(y)=\mathscr{L}_{1}(-y)$ for all $y \in I_{2}$ in the second case. Since $\mathscr{L}_{1}(y) \neq 0$ for all $y \in\left(0, e_{1}^{+}\right)$by assumption and $\mathscr{L}_{1}\left(\sigma_{1}(y)\right)=\mathscr{L}_{1}(y)$ for all $y \in I_{1}$, this shows that the Wronskian does not vanish on $\left(0, e_{2}^{+}\right)$. Accordingly we can apply Theorem 4.8 and assert that $\left(I_{0}, L\right)$ is indeed an ECT-system on $\left(0, h_{0}\right)$. This shows the claim and concludes the proof of the result.

We give next an example of a center with a monotonous period function for which the sufficient condition given in Proposition 3.2 is not fulfilled.

Example 4.12. Consider now $H(x, y)=F(x)+F(y)$ with $F(z)=\frac{1}{2} z^{2}+z^{4}+z^{6}+z^{8}$. In this case one can readily show that the center at the origin is global. Furthermore, by applying Lemma 2.4, the period function $T(h)$ is decreasing near $h=0$ since $\Delta=-4$. We will show that the period function is globally monotonous decreasing. To this end one can first try to apply Proposition 3.2, which requires

$$
\left(\frac{F_{i}}{\left(F_{i}^{\prime}\right)^{2}}\right)^{\prime \prime}(x)=6 \frac{-1+6 x^{2}+57 x^{4}+328 x^{6}+928 x^{8}+1592 x^{10}+1476 x^{12}+960 x^{14}+448 x^{16}}{\left(1+4 x^{2}+6 x^{4}+8 x^{6}\right)^{4}}
$$

to be non-vanishing on $(0,+\infty)$. By applying Sturm's Theorem it turns out however that this function has one positive root counted with multiplicities. Thus Proposition 3.2 does not apply but the desired result will follow by Theorem 4.11. Indeed, on account of $\sigma_{1}=-I d$, the assumption in $(a)$ is fulfilled, whereas the condition in $(b)$ writes as $\left(x F_{1}^{\prime}(x)\right)^{\prime}=2 x+16 x^{3}+36 x^{5}+64 x^{7} \neq 0$ for all $x>0$, which is obvious. Hence the period function has at most one critical period counting multiplicities. Finally we can discard the existence of one critical period by noting that, thanks to $(b)$ in Theorem $5.1, \lim _{h \rightarrow+\infty} T(h)=0$.

Finally we particularize Theorem 4.11 assuming additionally that $F$ is an even function.

Corollary 4.13. Assume that $F_{1}$ is even and $F_{2}=F_{1}$. If $\left(\frac{F_{1}}{\left(F_{1}^{\prime}\right)^{2}}\right)^{\prime \prime}$ has at most one zero on $\left(0, e_{1}^{+}\right)$, counted with multiplicities, and $\left(x F_{1}^{\prime}(x)\right)^{\prime}$ does not vanish on $\left(0, e_{1}^{+}\right)$, then the period function of the center at the origin of system (1) has at most one critical period, multiplicities taking into account.

Proof. The result will follow by applying Theorem 4.11. To this aim note that $\mathscr{P}_{\sigma_{1}}\left(\left(\frac{F_{1}}{\left(F_{1}^{\prime}\right)^{2}}\right)^{\prime \prime}\right)=\left(\frac{F_{1}}{\left(F_{1}^{\prime}\right)^{2}}\right)^{\prime \prime}$ because $F_{1}$ is an even function and $\sigma_{1}=-I d$. Hence $\mathscr{P}_{\sigma_{1}}\left(\left(\frac{F_{1}}{\left(F_{1}^{\prime}\right)^{2}}\right)^{\prime \prime}\right)$ has exactly one zero on $\left(0, e_{1}^{+}\right)$, counted with multiplicities, thanks to the first assumption in the statement. On the other hand, using again that $F_{1}$ is even, it follows that the condition $\left(\mathscr{P}_{\sigma_{1}}(1)(x)\right)^{2}+\mathscr{P}_{\sigma_{1}}\left(\frac{F_{1}^{\prime \prime}}{\left(F_{1}^{\prime}\right)^{2}}\right)(x) \mathscr{P}_{\sigma_{1}}\left(x F_{1}^{\prime}(x)\right) \neq 0$ for all $x \in\left(0, e_{1}^{+}\right)$simply writes as $\left(x F_{1}^{\prime}(x)\right)^{\prime} \neq 0$ for all $x \in\left(0, e_{1}^{+}\right)$, which is the second assumption in the statement. Hence Theorem 4.11 shows the validity of the result.

\section{$5 \quad$ Asymptotic results for the period function}

In this section, motivated by the tools employed by Kaplan and Yorke [13], we study the class of Hamiltonian differential systems with separable variables (1) such that $F_{1}(x)=F_{2}(-x)$. In other words, getting rid of the subscripts that are unnecessary in this case and setting $F^{\prime}=f$, we consider planar Hamiltonian differential systems of the form

$$
\left\{\begin{array}{l}
\dot{x}=-f(y), \\
\dot{y}=-f(-x) .
\end{array}\right.
$$

This type of differential system is related with differential-delay equations because of the following result by Kapplan and Yorke [13, Theorem 1.1]. 
Theorem (Kaplan-Yorke). Suppose that $f: \mathbb{R} \longrightarrow \mathbb{R}$ is a continuous and odd function verifying $x f(x)>0$ for all $x \neq 0$. Assume that $\lim _{x \rightarrow 0} \frac{f(x)}{x}=\alpha$ and $\lim _{x \rightarrow \infty} \frac{f(x)}{x}=\beta$ exist (allowing either $\alpha$ or $\beta$ to be 0 or $\infty)$. Finally, suppose that $\lim _{x \rightarrow \infty} F(x)=\infty$. Then the differential-delay equation $\dot{x}(t)=-f(x(t-1))$ has a nontrivial periodic solution $x(t)$ of period four if either $\alpha<\frac{\pi}{2}<\beta$ or $\beta<\frac{\pi}{2}<\alpha$. Furthermore, if $y(t)$ is defined to be $x(t-1)$, this periodic solution satisfies (5).

This seminal result, that goes back to 1973, was generalized and extended by Nussbaum (see [22, 23, 24]) without the hypothesis that $f$ is odd and $\lim _{x \rightarrow \infty} F(x)=\infty$. (The approach in this series of papers is completely different to the one in [13] because it relies in very sophisticated fixed point theorems in Banach spaces). As a matter of fact our interest on the issue arises from the proof of the result by Kaplan and Yorke rather than the result itself. This is so because their hypothesis imply that system (5) has a global center at the origin and the proof consists in proving the existence of a periodic orbit of period 4 . To this end they show that the period function of the center verifies $\lim _{h \rightarrow 0^{+}} T(h)=\frac{2 \pi}{\alpha}$ and $\lim _{h \rightarrow+\infty} T(h)=\frac{2 \pi}{\beta}$, and then the result follows by the intermediate value theorem. Our aim in this section is to study the limit of $T(h)$ at the endpoints of its domain without assuming $\lim _{x \rightarrow \infty} F(x)=\infty$ (which forces the center to be global) and that $f$ is odd. That being said, we point out that the regularity assumptions in this section, contrary to the rest of the paper, is that $f$ is merely continuous. In addition to this, the standing hypothesis will be that the limits

$$
\alpha:=\lim _{x \rightarrow 0} \frac{f(x)}{x}, \beta_{-}:=\lim _{x \rightarrow-\infty} \frac{f(x)}{x} \text { and } \beta_{+}:=\lim _{x \rightarrow+\infty} \frac{f(x)}{x}
$$

exist, allowing them to be zero or $+\infty$. Since $F^{\prime}=f$, the latter imply $\lim _{x \rightarrow \pm \infty} \frac{F(x)}{x^{2}}=\frac{1}{2} \beta_{ \pm}$by L'Hôpital's Rule (see [33]), so that $h_{ \pm}:=\lim _{x \rightarrow \pm \infty} F(x) \in \mathbb{R}_{>0} \cup\{+\infty\}$ exist. Let us set $H(x, y)=F(-x)+F(y)$ and recall that then $H(\mathscr{P})=\left(0, h_{0}\right)$, where $\mathscr{P}$ stands for the period annulus of the center at the origin of system (5). We are now in position to state our main result in this section.

Theorem 5.1. Suppose that $f$ is a continuous function on $\mathbb{R}$ satistying that the limits in (6) exist and that $x f(x)>0$ for all $x \neq 0$. Then the period $T(h)$ of the periodic orbit $\gamma_{h}$ of $(5)$ inside the energy level $H=h$ verifies the following properties:

(a)

$$
\lim _{h \rightarrow 0^{+}} T(h)=\left\{\begin{array}{cl}
\frac{2 \pi}{\alpha} & \text { if } \alpha>0 \\
+\infty & \text { if } \alpha=0 \\
0 & \text { if } \alpha=+\infty
\end{array}\right.
$$

(b) Assume that $\beta_{+}=+\infty$ and $\beta_{-}=+\infty$. Then the origin is a global center and $\lim _{h \rightarrow+\infty} T(h)=0$.

(c) Assume that $\beta_{+}<+\infty$ and $\beta_{-}=+\infty$ (respectively, $\beta_{+}=+\infty$ and $\beta_{-}<+\infty$ ).

$\left(c_{1}\right)$ If $h_{+}\left(\right.$respectively, $\left.h_{-}\right)$is finite then the center is non-global with $h_{0}=h_{+}\left(\right.$respectively, $\left.h_{0}=h_{-}\right)$ and $\lim _{h \rightarrow h_{0}^{-}} T(h)=+\infty$.

(c) If $h_{+}=+\infty$ (respectively, $\left.h_{-}=+\infty\right)$, then the center is global and $\lim _{h \rightarrow+\infty} T(h)$ is equal to $\frac{\pi}{2 \beta_{+}}$ when $\beta_{+}>0$ and $+\infty$ when $\beta_{+}=0$ (respectively, $\frac{\pi}{2 \beta_{-}}$when $\beta_{-}>0$ and $+\infty$ when $\beta_{-}=0$ ).

(d) Assume that $\beta_{+}<+\infty$ and $\beta_{-}<+\infty$.

$\left(d_{1}\right)$ If $h_{+}$or $h_{-}$is finite then the center is non-global with $h_{0}=\min \left\{h_{+}, h_{-}\right\}$and $\lim _{h \rightarrow h_{0}^{-}} T(h)=+\infty$.

$\left(d_{2}\right)$ If $h_{+}=h_{-}=+\infty$, then the center is global, and

$$
\lim _{h \rightarrow+\infty} T(h)=\left\{\begin{array}{cl}
\frac{\pi}{2}\left(\frac{1}{\sqrt{\beta_{+}}}+\frac{1}{\sqrt{\beta_{-}}}\right)^{2} & \text { if } \beta_{ \pm} \in(0,+\infty), \\
+\infty & \text { if } \beta_{-}=0 \text { or } \beta_{+}=0 .
\end{array}\right.
$$


There are some previous results in the literature related with Theorem 5.1 that should be referred. As we already mentioned, Kaplan and Yorke show the assertions $(b)$ and $\left(d_{2}\right)$ in their proof of $[13$, Theorem 1.1] under the additional assumption that $f$ is odd. In this respect we refer the reader to Remark 5.2 for some further comments. On the other hand, [7, Theorem $\mathrm{C}$ ] gives the first term in the asymptotic expansion of $T(h)$ at $h=+\infty$ for general Hamiltonian differential systems with separable variables, and its application yields to the assertions in Theorem 5.1 with regard to the global center case, i.e., $(b),\left(c_{2}\right)$ and $\left(d_{2}\right)$. Finally the fact that $\lim _{h \rightarrow 0^{+}} T(h)$ can be given in terms of the linear part of the center is a classical result even for general differential systems and we include the assertion in $(a)$ for completeness.

Theorem 5.1 gives the limit of $T(h)$ as $h$ tends to the endpoints of its domain of definition $\left(0, h_{0}\right)$. Thus, in combination with the intermediate value theorem, it can be used to prove the existence of periodic orbits with prescribed periods. Likewise, taking the monotonicity of $T(h)$ near $h=0$ or $h=h_{0}$ into account, it can also be used to prove the existence of critical periodic orbits (see for example the proof of [3, Theorem A] or [16, Theorem 5.2]). Of course to this end it is necessary to compute the period constants (cf. Lemma 2.4), that give the monotonicity of $T(h)$ at $h=0$, or to study the asymptotic development of $T(h)$ at $h=h_{0}$, which constitutes a much more difficult problem (cf. [15, Theorem A]). It is also worth to remark that the proof of Theorem 5.1 shows that in cases $\left(c_{2}\right)$ and $\left(d_{2}\right)$ the periodic orbit $\gamma_{h}$ undergoes a kind of slow-fast phenomenon as $h$ tends to $+\infty$. Example 5.4 shows an explicit Hamiltonian differential system that exhibits this type of motion. Slow-fast oscillations occur typically in singular perturbation problems and so we think that it is an interesting issue for further research.

Proof of Theorem 5.1. On account of the hypothesis $x f(x)>0$ for all $x \in \mathbb{R} \backslash\{0\}$, the Hamiltonian verifies $H(x, y)>0$ for all $(x, y) \in \mathbb{R}^{2} \backslash\{(0,0)\}$ and the origin, which is a center, is the unique singular point of the differential system. Moreover the continuity of $f$ implies $H(0,0)=0$. As in the previous section we denote by $\mathscr{P}$ the period annulus of the center and by $\gamma_{h}$ the periodic orbit inside the energy level $H=h$. We also suppose that $H(\mathscr{P})=\left(0, h_{0}\right)$ with $h_{0} \in \mathbb{R}_{>0} \cup\{+\infty\}$.

An easy computation shows that the differential system (5) writes in polar coordinates as

$$
\left\{\begin{array}{l}
\dot{r}=R(r, \theta):=-\cos \theta f(r \sin \theta)-\sin \theta f(-r \cos \theta), \\
\dot{\theta}=\Theta(r, \theta):=(-\cos \theta f(-r \cos \theta)+\sin \theta f(r \sin \theta)) / r .
\end{array}\right.
$$

Observe that, due to $x f(x)>0$ for all $x \in \mathbb{R} \backslash\{0\}, \Theta(r, \theta)>0$ for all $\theta$ and $r>0$. It is well known that this permits to express the radius of any solution of (5) in terms of the angle. Indeed, to this end we consider

$$
\frac{d r}{d \theta}=\frac{R(r, \theta)}{\Theta(r, \theta)}
$$

For convenience (in order to take advantage of the symmetry), let $r(\theta ; h)$ be the solution of this differential equation with initial condition $r(-\pi / 4 ; h)=r_{h}$ where $r_{h}$ is defined implicitly by means of $H\left(\frac{r_{h}}{\sqrt{2}},-\frac{r_{h}}{\sqrt{2}}\right)=h$. Then, taking advantage of the symmetry of system (5) with respect to $y=-x$, we can express the period of the periodic orbit $\gamma_{h}$ as the integral

$$
T(h)=2 \int_{-\frac{\pi}{4}}^{\frac{3 \pi}{4}} \frac{d \theta}{\Theta(r(\theta ; h), \theta)} .
$$

Furthermore it is clear that $T(h)=2\left(T_{1}(h)+T_{2}(h)+T_{3}(h)\right)$, where

$$
T_{1}(h):=\int_{-\frac{\pi}{4}}^{0} \frac{d \theta}{\Theta(r(\theta ; h), \theta)}, T_{2}(h):=\int_{0}^{\frac{\pi}{2}} \frac{d \theta}{\Theta(r(\theta ; h), \theta)} \text { and } T_{3}(h):=\int_{\frac{\pi}{2}}^{\frac{3 \pi}{4}} \frac{d \theta}{\Theta(r(\theta ; h), \theta)} .
$$

We now proceed to prove separately each assertion in the statement. 
(a) By the continuity of $f$ at zero we have that $\lim _{h \rightarrow 0^{+}} r(\theta ; h)=0$ uniformly in $\theta$. Thus, since

$$
\Theta(r, h)=\cos ^{2} \theta \frac{f(-r \cos \theta)}{-r \cos \theta}+\sin ^{2} \theta \frac{f(r \sin \theta)}{r \sin \theta},
$$

the hypothesis $\lim _{x \rightarrow 0} \frac{f(x)}{x}=\alpha$ implies that $\lim _{h \rightarrow 0^{+}} \Theta(r(\theta ; h), \theta)=\alpha$ uniformly in $\theta$. If $\alpha=0$ then by applying Fatou's Lemma (see [28, Theorem 11.31] for instance) we get

$$
\lim _{h \rightarrow 0^{+}} T(h) \geqslant 2 \int_{-\frac{\pi}{4}}^{\frac{3 \pi}{4}} \lim _{h \rightarrow 0^{+}}\left(\frac{1}{\Theta(r(\theta ; h), \theta)}\right) d \theta=+\infty .
$$

If $\alpha \in \mathbb{R}_{>0} \cup\{+\infty\}$ then there exist $\varepsilon>0$ and $K>0$ such that $\Theta(r(\theta ; h), \theta)>K$ for all $\theta$ and $r \in(0, \varepsilon)$. Consequently the integrand in $T(h)$ is uniformly bounded by a constant and the application of the Lebesgue's Dominated Convergence Theorem (see [28, Theorem 11.32] for instance) gives

$$
\lim _{h \rightarrow 0^{+}} T(h)=2 \int_{-\frac{\pi}{4}}^{\frac{3 \pi}{4}} \lim _{h \rightarrow 0^{+}}\left(\frac{1}{\Theta(r(\theta ; h), \theta)}\right) d \theta=\left\{\begin{array}{cl}
\frac{2 \pi}{\alpha} & \text { if } \alpha \in \mathbb{R}_{>0}, \\
0 & \text { if } \alpha=+\infty
\end{array}\right.
$$

as desired.

(b) Note first that the hypothesis $\beta_{ \pm}=+\infty$ implies $\lim _{x \rightarrow \pm \infty} F(x)=+\infty$, which in turn implies that the center is global with $h_{0}=+\infty$. Consequently, due to

$$
F(-r \cos \theta)+\left.F(r \sin \theta)\right|_{r=r(\theta ; h)}=h \text { for all } \theta \text { and } h>0,
$$

we have that $r(\theta ; h) \rightarrow+\infty$ as $h \rightarrow+\infty$ and $\theta \rightarrow \theta_{0}$ for any $\theta_{0}$. Hence $\lim _{h \rightarrow+\infty} r(\theta ; h)=+\infty$ uniformly in $\theta$. This shows, on account of the lower bounds

$$
\Theta(r, \theta) \geqslant \cos ^{2} \theta \frac{f(-r \cos \theta)}{-r \cos \theta} \geqslant \frac{1}{2} \frac{f(-r \cos \theta)}{-r \cos \theta} \text { for all } \theta \in\left[-\frac{\pi}{4}, \frac{\pi}{4}\right]
$$

and

$$
\Theta(r, \theta) \geqslant \sin ^{2} \theta \frac{f(r \sin \theta)}{r \sin \theta} \geqslant \frac{1}{2} \frac{f(r \sin \theta)}{r \sin \theta} \text { for all } \theta \in\left[\frac{\pi}{4}, \frac{3 \pi}{4}\right],
$$

together with the assumptions $\lim _{x \rightarrow \pm \infty} \frac{f(x)}{x}=+\infty$, that there exist two positive real numbers $k$ and $M$ such that $\Theta(r(\theta ; h), \theta)>k$ for all $\theta \in\left[-\frac{\pi}{4}, \frac{3 \pi}{4}\right]$ and $h>M$. Exactly as before, since it is clear that

$$
\lim _{h \rightarrow+\infty} \Theta(r(\theta ; h), \theta)=+\infty \text { for all } \theta \in\left(-\frac{\pi}{4}, \frac{3 \pi}{4}\right),
$$

by applying the Lebesgue's Dominated Convergence Theorem we can assert that

$$
\lim _{h \rightarrow+\infty} T(h)=2 \int_{-\frac{\pi}{4}}^{\frac{3 \pi}{4}} \lim _{h \rightarrow+\infty}\left(\frac{1}{\Theta(r(\theta ; h), \theta)}\right) d \theta=0 .
$$

(c) Let us consider for instance the case $\beta_{+}<+\infty$ and $\beta_{-}=+\infty$ (the other one follows verbatim). Note in this case that $\beta_{-}=+\infty$ implies $h_{-}=\lim _{x \rightarrow-\infty} F(x)=+\infty$. With regard to the behaviour of $F$ at $+\infty$ there are two possibilities to consider:

$\left(c_{1}\right) h_{+}=\lim _{x \rightarrow+\infty} F(x)<+\infty$

Consider the set of periodic orbits $\left\{\gamma_{h}\right\}_{h \in\left(0, h_{0}\right)}$ inside the period annulus $\mathscr{P}$ and recall that each $\gamma_{h}$ is inside the level curve $H(x, y)=F(-x)+F(y)=h$. We claim that these level curves are ovals surrounding the origin provided that $h \in\left(0, h_{+}\right)$, i.e., that $H(\mathscr{P})=\left(0, h_{0}\right)$ with $h_{0}=h_{+}$. In order to show the claim note first that $F:(0,+\infty) \longrightarrow\left(0, h_{+}\right)$and $F:(-\infty, 0) \longrightarrow(0,+\infty)$ 
are diffeomorphisms because $x f(x)>0$ for all $x \neq 0$. Denote their inverses by $F_{+}^{-1}$ and $F_{-}^{-1}$, respectively. Then $F(-x)+F(y)=h$ gives two branches $y=y_{ \pm}(x ; h):=F_{ \pm}^{-1}(h-F(-x))$ with $y_{-}<0<y_{+}$. For each fixed $h \in\left(0, h_{+}\right)$the map $x \longmapsto y_{+}(x ; h)$ is well defined and continuous provided that $F(-x)<h$, i.e., for $x$ such that $x_{-}(h)<x<x_{+}(h)$ where $x_{ \pm}(h):=-F_{\mp}^{-1}(h)$. Furthermore $F(-x)+F\left(y_{+}(x ; h)\right)=h$ implies that $y_{+}(x ; h)$ tends to $+\infty$ as $(x, h) \rightarrow\left(0, h_{+}\right)$ because $F(0)=0$. Hence the level set $H=h_{+}$is unbounded. It is also clear that, for each $h \in\left(0, h_{+}\right)$, the level set $H=h$ is an oval surrounding the origin that can be parametrized by means of the graphs of $x \longmapsto y_{ \pm}(x ; h)$ with $x \in\left(x_{-}(h), x_{+}(h)\right)$. This proves the claim. What is more, the notation introduced so far enables to write the period of the periodic orbit $\gamma_{h}$ as

$$
T(h)=\int_{x_{-}(h)}^{x_{+}(h)} \frac{d x}{f\left(y_{+}(x ; h)\right)}+\int_{x_{+}(h)}^{x_{-}(h)} \frac{d x}{f\left(y_{-}(x ; h)\right)},
$$

where each summand is positive because $x f(x)>0$ for all $x \neq 0$. Taking $F(-x)+F\left(y_{ \pm}(x ; h)\right)=h$ and $\lim _{x \rightarrow+\infty} F(x)=h_{+}$into account, it follows that

$$
\lim y_{ \pm}(x ; h)=0 \text { as }(x, h) \rightarrow\left(-\infty, h_{+}\right) .
$$

Consequently, since $f$ is continuous and $f(0)=0$, there exist $x_{c}<0$ and $h_{c} \in\left(0, h_{+}\right)$such that $0<f\left(y_{+}(x ; h)\right)<1$ for all $x \in\left(x_{-}(h), x_{c}\right)$ with $h \in\left(h_{c}, h_{+}\right)$. Accordingly, if $h \in\left(h_{c}, h_{+}\right)$then

$$
T(h)>\int_{x_{-}(h)}^{x_{+}(h)} \frac{d x}{f\left(y_{+}(x ; h)\right)}>\int_{x_{-}(h)}^{x_{c}} \frac{d x}{f\left(y_{+}(x ; h)\right)}>x_{c}-x_{-}(h),
$$

and the result follows noting that $x_{-}(h)=-F_{+}^{-1}(h)$ tends to $-\infty$ as $h \rightarrow h_{+}$.

$\left(c_{2}\right) h_{+}=\lim _{x \rightarrow+\infty} F(x)=+\infty$

In this subscase we have $h_{+}=h_{-}=+\infty$ and so the center is global. Acordingly, exactly as we argue in $(b), \lim _{h \rightarrow+\infty} r(\theta ; h)=+\infty$ uniformly in $\theta$.

Let us discuss the case $\beta_{+}>0$ first. Then, on account of the hypothesis $\lim _{x \rightarrow+\infty} \frac{f(x)}{x}=\beta_{+}$and the lower bound

$$
\Theta(r, \theta) \geqslant \sin ^{2} \theta \frac{f(r \sin \theta)}{r \sin \theta} \geqslant \frac{1}{2} \frac{f(r \sin \theta)}{r \sin \theta} \text { for all } \theta \in\left[\frac{\pi}{4}, \frac{3 \pi}{4}\right],
$$

there exists $M_{+}>0$ such that $\Theta(r, \theta) \geqslant \frac{1}{4} \beta_{+}$for all $\theta \in\left[\frac{\pi}{4}, \frac{3 \pi}{4}\right]$ and $r>M_{+}$. Similarly, due to

$$
\Theta(r, \theta) \geqslant \cos ^{2} \theta \frac{f(-r \cos \theta)}{-r \cos \theta} \geqslant \frac{1}{2} \frac{f(-r \cos \theta)}{-r \cos \theta} \text { for all } \theta \in\left[-\frac{\pi}{4}, \frac{\pi}{4}\right],
$$

and the assumption $\lim _{x \rightarrow-\infty} \frac{f(x)}{x}=+\infty$, there exist two positive numbers $k$ and $M_{-}$such that $\Theta(r, \theta)>k$ for all $\theta \in\left[-\frac{\pi}{4}, \frac{\pi}{4}\right]$ and $r>M_{-}$. Then, using these two lower bounds together with the fact that $\lim _{h \rightarrow+\infty} r(\theta ; h)=+\infty$ uniformly in $\theta$, we can assert that there exist $\ell, M>0$ such that $\Theta(r(\theta ; h), \theta)>\ell$ for all $\theta \in\left[-\frac{\pi}{4}, \frac{3 \pi}{4}\right]$ and $r>M$. This gives a constant uniform upper bound for the integrands in $T_{i}(h), i=1,2,3$, that enables to apply the Lebesgue's Dominated Convergence Theorem once again. In doing so, since $\lim _{h \rightarrow+\infty} \Theta(r(\theta ; h), \theta)=0$ for all $\theta \in\left(-\frac{\pi}{4}, \frac{\pi}{2}\right)$ due to $\beta_{-}=+\infty$, we get that $\lim _{h \rightarrow+\infty} T_{1}(h)=\lim _{h \rightarrow+\infty} T_{2}(h)=0$. Furthermore $\lim _{h \rightarrow+\infty} T_{3}(h)=\frac{\pi}{4 \beta_{+}}$ because $\lim _{h \rightarrow+\infty} \Theta(r(\theta ; h), \theta)=\beta_{+}$for all $\theta \in\left(\frac{\pi}{2}, \frac{3 \pi}{4}\right)$. Therefore $T(h)=2\left(T_{1}(h)+T_{2}(h)+T_{3}(h)\right)$ tends to $\frac{\pi}{2 \beta_{+}}$as $h \rightarrow+\infty$. (In this case the periodic orbits $\gamma_{h}$ exhibit a kind of slow-fast motion as $h$ tends to $+\infty$ because $T_{3}(h)$ tends to $\frac{\pi}{4 \beta_{+}}$, whereas $T_{1}(h)$ and $T_{2}(h)$ tend to zero.)

The case $\beta_{+}=0$ is shorter because, by Fatou's Lemma, $\lim _{h \rightarrow+\infty} T_{3}(h)=+\infty$, which clearly suffices to show the validity of the assertion since $T_{1}$ and $T_{2}$ are positive.

(d) Let us treat finally the case in which $\beta_{+}$and $\beta_{-}$are both finite. To this end it suffices to consider the following two cases. 
$\left(d_{1}\right) h_{+}$or $h_{-}$is finite:

If $h_{+} \leqslant h_{-}$(which in particular implies $h_{+}<+\infty$ ) then the result follows verbatim the proof of $\left(c_{1}\right)$ because in that case, although we assume $h_{-}=+\infty$, it is only used that $h_{-} \geqslant h_{+}$. That being said, if $h_{-} \leqslant h_{+}$then one can reduce to the previous case by means of the change $(x, y) \longmapsto(-x,-y)$. and considering $F \circ(-I d)$ instead of $F$.

$\left(d_{2}\right) h_{+}=h_{-}=+\infty$ :

In this case the center is global, $H(\mathscr{P})=(0,+\infty)$ and, exactly as we argue in $(b)$, it follows that $\lim _{h \rightarrow+\infty} r(\theta ; h)=+\infty$ uniformly in $\theta$. Taking this into account we proceed with the proof by distinguishing the three cases that can occur:

1. $\left\{\beta_{+}=0\right.$ and $\left.\beta_{-}=0\right\}$

Consequently, recall (7), $\lim _{x \rightarrow \pm \infty} \frac{f(x)}{x}=\beta_{ \pm}=0$ implies that $\lim _{h \rightarrow+\infty} \Theta(r(\theta ; h), \theta)=+\infty$ for each fixed $\theta$. Then, by Fatou's Lemma,

$$
\lim _{h \rightarrow+\infty} T(h) \geqslant 2 \int_{-\frac{\pi}{4}}^{\frac{3 \pi}{4}} \lim _{h \rightarrow+\infty}\left(\frac{1}{\Theta(r(\theta ; h), \theta)}\right) d \theta=+\infty .
$$

2. $\left\{\beta_{+}>0\right.$ and $\left.\beta_{-}>0\right\}$

Thus, thanks to the hypothesis $\lim _{x \rightarrow+\infty} \frac{f(x)}{x}=\beta_{+}$and the lower bound

$$
\Theta(r, \theta) \geqslant \sin ^{2} \theta \frac{f(r \sin \theta)}{r \sin \theta} \geqslant \frac{1}{2} \frac{f(r \sin \theta)}{r \sin \theta} \text { for all } \theta \in\left[\frac{\pi}{4}, \frac{3 \pi}{4}\right],
$$

there exists $M_{+}>0$ such that $\Theta(r, \theta) \geqslant \frac{1}{4} \beta_{+}$for all $\theta \in\left[\frac{\pi}{4}, \frac{3 \pi}{4}\right]$ and $r>M_{+}$. Likewise,

$$
\Theta(r, \theta) \geqslant \cos ^{2} \theta \frac{f(-r \cos \theta)}{-r \cos \theta} \geqslant \frac{1}{2} \frac{f(-r \cos \theta)}{-r \cos \theta} \text { for all } \theta \in\left[-\frac{\pi}{4}, \frac{\pi}{4}\right]
$$

and $\lim _{x \rightarrow-\infty} \frac{f(x)}{x}=\beta_{-}$, imply the existence of $M_{-}>0$ satisfying that $\Theta(r, \theta) \geqslant \frac{1}{4} \beta_{-}$for all $\theta \in\left[-\frac{\pi}{4}, \frac{\pi}{4}\right]$ and $r>M_{-}$. Hence there exists $M>0$ so that $\Theta(r(\theta ; h), \theta) \geqslant \frac{1}{4} \beta_{m}$ for all $h>M$ and $\theta \in\left[-\frac{\pi}{4}, \frac{3 \pi}{4}\right]$. For each $i=1,2,3$, this provides a constant uniform upper bound for the integrand in $T_{i}(h)$, which enables to apply the Lebesgue's Dominated Convergence Theorem to compute $\lim _{h \rightarrow+\infty} T_{i}(h)$. In doing so, since $\lim _{h \rightarrow+\infty} \Theta(r(\theta ; h), \theta)=\beta_{-}$for each $\theta \in\left(-\frac{\pi}{4}, 0\right)$, it turns out $\lim _{h \rightarrow+\infty} T_{1}(h)=\frac{\pi}{4 \beta_{-}}$. Similarly $\lim _{h \rightarrow+\infty} \Theta(r(\theta ; h), \theta)=\beta_{+}$for each $\theta \in\left(\frac{\pi}{2}, \frac{3 \pi}{4}\right)$ implies $\lim _{h \rightarrow+\infty} T_{3}(h)=\frac{\pi}{4 \beta_{+}}$. Finally, since $\lim _{h \rightarrow+\infty} \Theta(r(\theta ; h), \theta)=\beta_{-} \cos ^{2} \theta+\beta_{+} \sin ^{2} \theta$ for each $\theta \in\left(0, \frac{\pi}{2}\right)$ and, on the other hand,

$$
\int_{0}^{\frac{\pi}{2}} \frac{d \theta}{\beta_{-} \cos ^{2} \theta+\beta_{+} \sin ^{2} \theta}=\frac{\pi}{2 \sqrt{\beta_{+} \beta_{-}}},
$$

we get that $\lim _{h \rightarrow+\infty} T_{2}(h)=\frac{\pi}{2 \sqrt{\beta_{+} \beta_{-}}}$. Therefore, taking $T=2\left(T_{1}+T_{2}+T_{3}\right)$ into account,

$$
\lim _{h \rightarrow+\infty} T(h)=\frac{\pi}{2}\left(\frac{1}{\beta_{-}}+\frac{2}{\sqrt{\beta_{+} \beta_{-}}}+\frac{1}{\beta_{+}}\right)=\frac{\pi}{2}\left(\frac{1}{\sqrt{\beta_{+}}}+\frac{1}{\sqrt{\beta_{-}}}\right)^{2} .
$$

3. $\left\{\beta_{-}=0\right.$ and $\left.\beta_{+}>0\right\}$ or $\left\{\beta_{-}>0\right.$ and $\left.\beta_{+}=0\right\}$

Let us consider the first case since the second one follows similarly. Then, exactly as we did in the study of the preceding cases, $\lim _{h \rightarrow+\infty} T(h) \geqslant \lim _{h \rightarrow+\infty} T_{1}(h)=+\infty$ by Fatou's Lemma. (In contrast to $\lim _{h \rightarrow+\infty} T_{1}(h)=+\infty$, the application of the Lebesgue's Dominated Convergence Theorem shows that $\lim _{h \rightarrow+\infty} T_{3}(h)=\frac{\pi}{4 \beta_{+}}$. Hence this is another case in which the periodic orbits $\gamma_{h}$ undergo a slow-fast phenomenon as $h \rightarrow+\infty$.) 


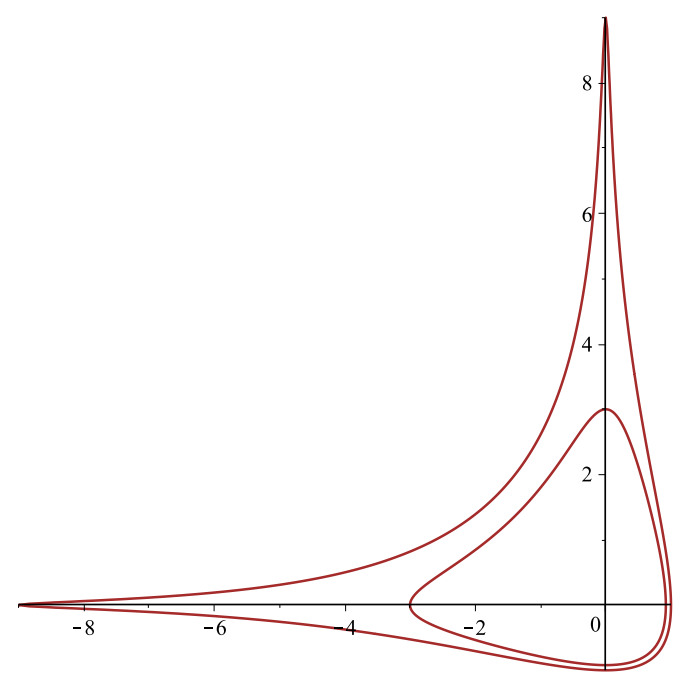

Figure 1: Level curves for the Hamiltonian function considered in Example 5.3.

This completes the proof of the result.

Remark 5.2. To prove their result about differential-delay equations stated at the beginning of the section, Kaplan and Yorke show that if $f$ is an odd function (which implies $\beta_{+}=\beta_{-}$) and $h_{ \pm}=+\infty$ (which implies that the center is global) then $\lim _{h \rightarrow+\infty} T(h)=\frac{2 \pi}{\beta_{ \pm}}$. Following our notation, their proof relies in the identity

$$
\int_{0}^{T(h)} \Theta(r(t ; h), \theta(t ; h)) d t=2 \pi \text { for all } h \in(0,+\infty),
$$

where $r(t ; h)$ and $\theta(t ; h)$ are respectively the radial and angular coordinates of the solution of the differential system (5) inside the energy level $H=h$. (This identity follows straighforward by taking $\frac{d \theta}{d t}=\Theta(r, \theta)$ into account.) Then, using that $\lim _{h \rightarrow+\infty} r(t ; h)=+\infty$ for each fixed $t$, together with the fact that

$$
\Theta(r, \theta)=\cos ^{2} \theta \frac{f(r \cos \theta)}{r \cos \theta}+\sin ^{2} \theta \frac{f(r \sin \theta)}{r \sin \theta} \longrightarrow \beta_{ \pm} \text {as } r \text { tends to }+\infty
$$

for each fixed $\theta \notin\left\{0, \frac{\pi}{2}, \pi, \frac{3 \pi}{2}\right\}$, they claim that by making $h \rightarrow+\infty$ in (8) one gets $\beta_{ \pm} \lim _{h \rightarrow+\infty} T(h)=2 \pi$. However this reasoning assumes the interchange of limit and integration, which is a delicate point.

Example 5.3. If we consider $H(x, y)=F(-x)+F(y)$ with $F(x)=1-e^{-x}(1+x)$ then we obtain

$$
\left\{\begin{array}{l}
\dot{x}=-y e^{-y} \\
\dot{y}=x e^{x}
\end{array}\right.
$$

which corresponds to the case $\left(c_{1}\right)$ in Theorem 5.1 because $\beta_{+}=0, \beta_{-}=+\infty, h_{+}=1$ and $h_{-}=+\infty$. Therefore the center is non-global with $h_{0}=h_{+}=1$ and $\lim _{h \rightarrow 1^{-}} T(h)=+\infty$. Figure 1 displays a numerical plot of the level curves $H(-3,0) \approx 0.801$ and $H(-9,0) \approx 0.999$.

Example 5.4. Likewise, the choice $F(x)=\frac{1}{2}\left(1+x^{2}-(1+x) e^{-x}\right)$ yields to the differential system

$$
\left\{\begin{array}{l}
\dot{x}=-y\left(1+e^{-y}\right) \\
\dot{y}=x\left(1+e^{x}\right)
\end{array}\right.
$$




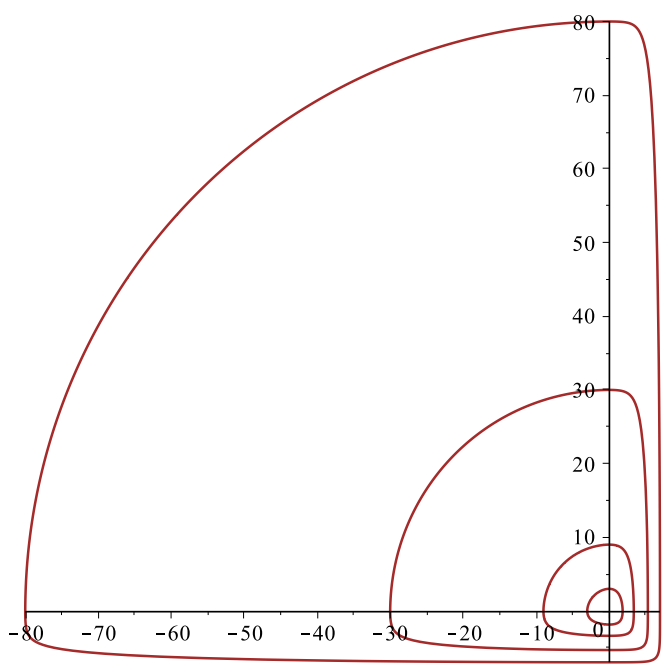

Figure 2: Level curves for the Hamiltonian function considered in Example 5.4.

It satisfies $\beta_{+}=1, \beta_{-}=+\infty$ and $h_{ \pm}=+\infty$, so that it corresponds to the case $\left(c_{2}\right)$ in Theorem 5.1. Thus the center is global and $\lim _{h \rightarrow+\infty} T(h)=\frac{\pi}{2}$, see Figure 2. Recall that to prove this case we split the period function as $T=2\left(T_{1}+T_{2}+T_{3}\right)$ and it occurs that $\lim _{h \rightarrow+\infty} T_{1}(h)=\lim _{h \rightarrow+\infty} T_{2}(h)=0$, whereas $\lim _{h \rightarrow+\infty} T_{3}(h)=\frac{\pi}{4 \beta_{+}}$. Therefore the intermediate time that spends $\gamma_{h}$ to cross the second quadrant tends to $\frac{\pi}{2 \beta_{+}}$as $h \rightarrow+\infty$, while the intermediate time to cross the other quadrants tends to zero. This kind of slow-fast phenomenon is certainly interesting to be studied.

Example 5.5. We give now two last examples for which the period function can be computed explicitly. The first one follows taking $F(x)=\frac{1}{4} x^{4}$, so that $\alpha=0$ and $\beta_{ \pm}=+\infty$. Some easy computations show that $T(h)=k h^{-1 / 2}$ with $k=\frac{8}{\sqrt{\pi}} \Gamma(5 / 4)^{2}$, where $\Gamma$ is the Gamma function. For the second one we choose $F(x)=\frac{3}{4} x^{4 / 3}$, so that $\alpha=+\infty$ and $\beta_{ \pm}=0$. In this case $T(h)=k h^{1 / 2}$ with $k=\frac{6}{\sqrt{3 \pi}} \Gamma(3 / 4)^{2}$.

\section{Acknowledgments}

The first author is partially supported by MTM2017-86795-C3-2-P. The second author is partially supported by NNSF of China grants 11671254 and 11871334. The first author would like to thank the School of Mathematical Sciences of the Shanghai Jiao Tong University in People's Republic of China for the kind hospitality during his research stay.

\section{References}

[1] T.R. Blows and N.G. Lloyd, The number of limit cycles of certain polynomial differential equations, Proc. Roy. Soc. Edinburgh Sect. A 98 (1984), 215-239.

[2] C. Chicone, The monotonicity of the period function for planar Hamiltonian vector fields, J. Differential Equations 69 (1987), 310-321. 
[3] C. Chicone and F. Dumortier, A quadratic system with a nonmonotonic period function, Proc. Amer. Math. Soc. 102 (1988) 706-710.

[4] C. Chicone and F. Dumortier, Finiteness for critical periods of planar analytic vector fields, Nonlinear Anal. 20 (1993) 315-335.

[5] C. Chicone and M. Jacobs, Bifurcation of critical periods for plane vector fields, Trans. Amer. Math. Soc. 312 (1989), 433-486.

[6] A. Cima, F. Mañosas and J. Villadelprat, Isochronicity for several classes of Hamiltonian systems, J. Differential Equations 157 (1999) 373-413.

[7] A. Cima, A. Gasull and F. Mañosas, Period function for a class of Hamiltonian systems, J. Differential Equations 168 (2000) 180-199.

[8] J.P. Françoise and C.C. Pugh, Keeping track of limit cycles, J. Differential Equations 65 (1986), 139157.

[9] E. Freire, A. Gasull and A. Guillamon, First derivative of the period function with applications, J. Differential Equations 204 (2004) 139-162.

[10] A. Garijo and J. Villadelprat, Algebraic and analytical tools for the study of the period function, J. Differential Equations 254 (2014) 2464-2484.

[11] M. Grau, F. Mañosas and J. Villadelprat, A Chebyshev criterion for Abelian integrals, Trans. Amer. Math. Soc. 363 (2011), 109-129.

[12] Y. Ilyashenko and S. Yakovenko, "Lectures on analytic differential equations" Graduate Studies in Mathematics, 86. American Mathematical Society, Providence, RI, 2008.

[13] J. Kaplan and J. Yorke, Ordinary differential equations which yield periodic solutions of differential delay equations, J. Math. Anal. Appl. 48 (1974) 317-324.

[14] S. Karlin and W. Studden, "Tchebycheff Systems: With Applications in Analysis and Statistics", Interscience Publishers, 1966.

[15] P. Mardešić, D. Marín and J. Villadelprat, On the time function of the Dulac map for families of meromorphic vector fields, Nonlinearity 16 (2003) 855-881.

[16] P. Mardešić, D. Marín and J. Villadelprat, The period function of reversible quadratic centers, J. Differential Equations 224 (2006) 120-171.

[17] P. Mardešić, L. Moser-Jauslin and C. Rousseau, Darboux linearization and isochronous centers with a rational first integral, J. Differential Equations 134 (1997), 216-268.

[18] P. Mardešić and M. Saavedra, Non-accumulation of critical points of the Poincar?time on hyperbolic polycycles, Proc. Amer. Math. Soc. 135 (2007) 3273-3282.

[19] F. Mañosas and J. Villadelprat, Area-preserving normalizations for centers of planar Hamiltonian systems, J. Differential Equations 179 (2002) 625-646.

[20] F. Mañosas and J. Villadelprat, Criteria to bound the number of critical periods, J. Differential Equations 246 (2009) 2415-2433.

[21] F. Mañosas and J. Villadelprat, Bounding the number of zeros of certain Abelian integrals, J. Differential Equations 251 (2011), 1656-1669. 
[22] R. Nussbaum, Periodic solutions of some nonlinear, autonomous functional differential equations. II. J. Differential Equations 14 (1973) 360-394.

[23] R. Nussbaum, Periodic solutions of special differential equations: an example in nonlinear functional analysis, Proc. Roy. Soc. Edinburgh Sect. A 81 (1978) 131-151.

[24] R. Nussbaum, Uniqueness and nonuniqueness for periodic solutions of $x^{\prime}(t)=-g(x(t-1))$, J. Differential Equations 34 (1979) 25-54.

[25] F. Rothe, Remarks on periods of planar Hamiltonian systems, SIAM J. Math. Anal. 24 (1993) 129-154.

[26] R. Roussarie, Bifurcation of Planar Vector Fields and Hilbert's Sixteenth Problem, Progr. in Math., vol. 164, Birkhüser Verlag, Basel, 1998.

[27] C. Rousseau and B. Toni, Local bifurcations of critical periods in the reduced Kukles system, Can. J. Math. 49 (1997), 338-358.

[28] W. Rudin, "Principles of mathematical analysis" International Series in Pure and Applied Mathematics. McGraw-Hill Book Co., New York-Auckland-Düsseldorf, 1976.

[29] M. Sabatini, Period function's convexity for Hamiltonian centers with separable variables, Ann. Polon. Math. 85 (2005) 153-163.

[30] M. Sabatini, The period functions' higher order derivatives, J. Differential Equations 253 (2012) 28252845.

[31] R. Schaaf, A class of Hamiltonian systems with increasing periods, J. Reine Angew. Math. 363 (1985) 96-109.

[32] R. Schaaf, "Global solution branches of two-point boundary value problems", Lecture Notes in Mathematics, 1458. Springer-Verlag, Berlin, 1990.

[33] A. E. Taylor, "L'Hospital's Rule", The American Mathematical Monthly, 59 (1952) 20-24.

[34] Yan-Qian Ye et al., "Theory of limit cycles," Transl. Math. Monographs, vol. 66, Amer. Math. Soc., Providence, R.I., 1984. 